\title{
Cauchy problem for viscous shallow water equations with a term of capillarity
}

\author{
Boris Haspot *
}

\begin{abstract}
In this article, we consider the compressible Navier-Stokes equation with density dependent viscosity coefficients and a term of capillarity introduced by Coquel et al in [13. This model includes at the same time the barotropic Navier-Stokes equations with variable viscosity coefficients, shallow-water system and the model of Rohde in [36].

We first study the well-posedness of the model in critical regularity spaces with respect to the scaling of the associated equations. In a functional setting as close as possible to the physical energy spaces, we prove global existence of solutions close to a stable equilibrium, and local in time existence for solutions with general initial data. Uniqueness is also obtained.
\end{abstract}

\section{Introduction}

This paper is devoted to the Cauchy problem for the compressible Navier-Stokes equation with viscosity coefficients depending on the density and with a capillary term coming from the works of Coquel, Rohde and theirs collaborators in [13, [36]. Let $\rho$ and $u$ denote the density and the velocity of a compressible viscous fluid. As usual, $\rho$ is a non-negative function and $u$ is a vector valued function defined on $\mathbb{R}^{N}$. Then, the Navier-Stokes equation for compressible fluids endowed with internal capillarity introduced in [36] reads:

$(S W)\left\{\begin{array}{l}\partial_{t} \rho+\operatorname{div}(\rho u)=0, \\ \partial_{t}(\rho u)+\operatorname{div}(\rho u \otimes u)-\operatorname{div}(2 \mu(\rho) D u)-\nabla(\lambda(\rho) \operatorname{div} u)+\nabla P(\rho)=\kappa \rho \nabla D[\rho],\end{array}\right.$

supplemented by the initial condition:

$$
\rho_{/ t=0}=\rho_{0}, \quad \rho u_{/ t=0}=\rho_{0} u_{0}
$$

and:

$$
D[\rho]=\phi * \rho-\rho
$$

where $\phi$ is chosen so that:

$$
\phi \in L^{\infty}\left(\mathbb{R}^{N}\right) \cap C^{1}\left(\mathbb{R}^{N}\right) \cap W^{1,1}\left(\mathbb{R}^{N}\right), \quad \int_{\mathbb{R}^{N}} \phi(x) d x=1, \quad \phi \text { even, and } \phi \geq 0
$$

*Université Paris XII - Val de Marne 61, avenue du Général de Gaulle 94010 CRETEIL Cedex Téléphone : (33-1) 45171651 Télécopie : (33-1) 45171649 e-mail : haspot@univ-paris12.fr 
and where $P(\rho)$ denotes the pressure, $\mu$ and $\lambda$ are the two Lamé viscosity coefficients (they depend regularly on the density $\rho$ ) satisfying:

$$
\mu>0 \quad 2 \mu+N \lambda \geq 0 .
$$

( $\mu$ is sometimes called the shear viscosity of the fluid, while $\lambda$ is usually referred to as the second viscosity coefficient).

several physical models arise as a particular case of system $(S W)$ :

- when $\kappa=0(S W)$ represents compressible Navier-Stokes model with variable viscosity coefficients.

- when $\kappa=0$ and $\mu(\rho)=\rho, \lambda(\rho)=0, P(\rho)=\rho^{2}, N=2$ then $(S W)$ describes the system of shallow-water.

- when $\kappa=0$ and $\mu, \lambda$ are constant, $(S W)$ reduce to the Rohde model of chapter four.

One of the major difficulty of compressible fluid mechanics is to deal with the vacuum. The problem of existence of global solution in time for Navier-Stokes equations was addressed in one dimension for smooth enough data by Kazhikov and Shelukin in [31], and for discontinuous ones, but still with densities away from zero, by Serre in [38] and Hoff in 24]. Those results have been generalized to higher dimension by Matsumura and Nishida in 33 . for smooth data close to equilibrium and by Hoff in the case of discontinuous data in [26, 27].

Concerning large initial data, Lions showed in 32 the global existence of weak solutions for $\gamma \geq \frac{3}{2}$ for $N=2$ and $\gamma \geq \frac{9}{5}$ for $N=3$. Let us mention that Feireisl in [20] generalized the result to $\gamma>\frac{N}{2}$ in establishing that we can obtain renormalized solution without imposing that $\rho \in L_{l o c}^{2}$, for this he introduces the concept of oscillation defect measure evaluating the loss of compactness.

Other results provide the full range $\gamma>1$ under symmetries assumptions on the initial datum, see Jiang and Zhang [29]. All those results do not require to be far from the vacuum. However they rely strongly on the assumption that the viscosity coefficients are bounded below by a positive constant. This non physical assumption allows to get some estimates on the gradient of the velocity field.

The main difficulty when dealing with vanishing viscosity coefficients on vacuum is that the velocity cannot even be defined when the density vanishes and so we cannot use some properties of parabolicity of the momentum equation, see [11], [12].

The first result handling this difficulty is due to Bresch, Desjardins and Lin in [7]. They show the existence of global weak solution for Korteweg system in choosing specific type of viscosity where $\mu$ and $\lambda$ are linked.

The result was later improved by Bresch and Desjardins in 4 to include the case of vanishing capillarity $(\kappa=0)$, but with an additional quadratic friction term $r \rho u|u|$ (see also [6]). However, those estimates are not enough to treat the case without capillarity and friction effects $\kappa=0$ and $r=0$ (which corresponds to equation (1) with $h(\rho)=\rho$ and $g(\rho)=0)$.

The main difficulty, to prove the stability of $(S W)$, is to pass to the limit in the term $\rho u \otimes u$ (which requires the strong convergence of $\sqrt{\rho} u$ ). Note that this is easy when the 
viscosity coefficients are bounded below by a positive constant. On the other hand, the new bounds on the gradient of the density make the control of the pressure term far simpler than in the case of constant viscosity coefficients.

In [6] Bresch and Desjardins show a result of global existence of weak solution for the non isothermal Navier-Stokes equation by imposing some condition between the viscosity coefficient and a bound by below on the viscosity coefficient. A. Mellet and A. Vasseur in using the same entropy inequality get a very interesting result of stability. They get more general estimate, which hold for any viscosity coefficients $\mu(\rho), \lambda(\rho)$ satisfying the relation:

$$
\mu(\rho)=\rho \lambda^{\prime}(\rho)-\lambda(\rho) .
$$

Mellet and Vasseur show in 34 the $L^{1}$ stability of weak solutions of Navier-Stokes compressible isotherm under some conditions on the viscosity coefficients (including (1.1)) but without any additional regularizing terms. The interest of this result is to consider conditions where the viscosity coefficients vanish on the vacuum set. It includes the case $\mu(\rho)=\rho, \lambda(\rho)=0$ (when $N=2$ and $\gamma=2$, where we recover the Saint-Venant model for Shallow water). The key to the proof is a new energy inequality on the velocity and a gain of integrability, which allows to pass to the limit.

The existence and uniqueness of local classical solutions for $(S W)$ with smooth initial data such that the density $\rho_{0}$ is bounded and bounded away from zero (i.e., $0<\underline{\rho} \leq \rho_{0} \leq M$ ) has been stated by Nash in [35. Let us emphasize that no stability condition was required there.

On the other hand, for small smooth perturbations of a stable equilibrium with constant positive density, global well-posedness has been proved in [33. Many recent works have been devoted to the qualitative behavior of solutions for large time (see for example [24, 31]). Refined functional analysis has been used for the last decades, ranging from Sobolev, Besov, Lorentz and Triebel spaces to describe the regularity and long time behavior of solutions to the compressible model [39], [40, 25], 30]. The most important result on the system of Navier-Stokes compressible isothermal comes from R. Danchin in [15] and [18] who show the existence of global solution and uniqueness with initial data close from a equilibrium, and he has the same result in finite time. The interest is that he can work in critical Besov space (critical in the sense of the scaling of the equation.) We generalize the result of $\mathrm{R}$. Danchin in considering general viscosity coefficient and in connecting this result with those of A. Mellet and A. Vasseur. This result improves too the case of strong solution for the shallow-water system, where W.Wang and C-J Xu in [41] have got global existence in time for small initial data with $h_{0}, u_{0} \in H^{2+s}$ with $s>0$.

\subsection{Notations and main results}

We will mainly consider the global well-posedness problem for initial data close enough to stable equilibria. Here we want to investigate the well-posedness of the system $(S W)$ problem in critical spaces, that is, in spaces which are invariant by the scaling of the equations. Let us explain precisely the scaling of the system. We can easily check that, if $(\rho, u)$ solves $(S W)$, so does $\left(\rho_{\lambda}, u_{\lambda}\right)$, where:

$$
\rho_{\lambda}(t, x)=\rho\left(\lambda^{2} t, \lambda x\right) \text { and } u_{\lambda}(t, x)=\lambda u\left(\lambda^{2} t, \lambda x\right)
$$


provided the pressure law $P$ has been changed into $\lambda^{2} P$.

Definition 1.1 We say that a functional space is critical with respect to the scaling of the equation if the associated norm is invariant under the transformation:

$$
(\rho, u) \longrightarrow\left(\rho_{\lambda}, u_{\lambda}\right)
$$

(up to a constant independent of $\lambda$ ).

This suggests us to choose initial data $\left(\rho_{0}, u_{0}\right)$ in spaces whose norm is invariant for all $\lambda>0$ by $\left(\rho_{0}, u_{0}\right) \longrightarrow\left(\rho_{0}(\lambda \cdot), \lambda u_{0}(\lambda \cdot)\right)$.

A natural candidate is the homogeneous Sobolev space $\dot{H}^{N / 2} \times\left(\dot{H}^{N / 2-1}\right)^{N}$, but since $\dot{H}^{N / 2}$ is not included in $L^{\infty}$, we cannot expect to get $L^{\infty}$ control on the density when $\rho_{0} \in \dot{H}^{N / 2}$.

This is the reason why as in the chapter two, instead of the classical homogeneous Sobolev space, we will consider homogeneous Besov spaces $B_{2,1}^{N / 2} \times\left(B_{2,1}^{N / 2-1}\right)^{N}$ with the same derivative index. This allows to control the density from below and from above, without requiring more regularity on derivatives of $\rho$. In the sequel, we will need to control the vacuum, this motivates the following definition:

Definition 1.2 Let $\bar{\rho}>0, \bar{\theta}>0$. We will note in the sequel:

$$
q=\frac{\rho-\bar{\rho}}{\bar{\rho}}
$$

Let us first state a result of global existence and uniqueness of $(S W)$ for initial data close to a equilibrium.

Theorem 1.1 Let $N \geq 2$. Let $\bar{\rho}>0$ be such that: $P^{\prime}(\bar{\rho})>0, \mu(\bar{\rho})>0$ and $2 \mu(\bar{\rho})+$ $\lambda(\bar{\rho})>0$. There exist two positive constants $\varepsilon_{0}$ and $M$ such that if $q_{0} \in \widetilde{B}^{\frac{N}{2}-1, \frac{N}{2}}, u_{0} \in$ $B^{\frac{N}{2}-1}$ and:

$$
\left\|q_{0}\right\|_{\widetilde{B}^{\frac{N}{2}-1, \frac{N}{2}}}+\left\|u_{0}\right\|_{B^{\frac{N}{2}-1}} \leq \varepsilon_{0}
$$

then $(S W)$ has a unique global solution $(q, u)$ in $E^{\frac{N}{2}}$ which satisfies:

$$
\|(q, u)\|_{E^{\frac{N}{2}}} \leq M\left(\left\|q_{0}\right\|_{\widetilde{B}^{\frac{N}{2}-1, \frac{N}{2}}}+\left\|u_{0}\right\|_{B^{\frac{N}{2}-1}}\right),
$$

for some $M$ independent of the initial data where:

$$
\begin{aligned}
E^{\frac{N}{2}}=\left[C_{b}\left(\mathbb{R}^{+}, \widetilde{B}^{\frac{N}{2}-1, \frac{N}{2}}\right) \cap L^{1}\left(\mathbb{R}^{+}, \widetilde{B}^{\frac{N}{2}+1, \frac{N}{2}}\right)\right] \times\left[C_{b}\left(\mathbb{R}_{+}, B^{\frac{N}{2}-1}\right)^{N}\right. & \\
& \left.\cap L^{1}\left(\mathbb{R}^{+}, B^{\frac{N}{2}+1}\right)^{N}\right] .
\end{aligned}
$$

In the following theorems, we want to show some result of existence and uniqueness in finite time for large initial velocity and initial density close to some constant.

The following result shows the existence and uniqueness in finite time with initial data in critical Besov space for the scaling of the equations. However as we said, we need for some technical reasons of an hypothesis of smallness on $q_{0}$. We note that we work on Besov space $B_{p}^{s}$ with general index $p$ on the integrability. 
Theorem 1.2 Let $p \in\left[1,+\infty\left[\right.\right.$. Let $q_{0} \in B_{p}^{\frac{N}{p}}$ and $u_{0} \in B_{p}^{\frac{N}{p}-1}$. Assume also that: $\left\|q_{0}\right\|_{B_{p}^{\frac{N}{p}}} \leq \varepsilon$ for a suitably small positive constant $\varepsilon>0$.

Under the assumptions of the theorem 1.1 for the physical coefficients, there exists a time $T>0$ such that the following results hold:

1. Existence: If $p \in\left[1,2 N\left[\right.\right.$ then system $(S W)$ has a solution $(q, u)$ in $F_{p}^{\frac{N}{p}}$ with:

$$
F_{p}^{\frac{N}{p}}=\widetilde{C}_{T}\left(B_{p}^{\frac{N}{p}}\right) \times\left(L_{T}^{1}\left(B_{p}^{\frac{N}{p}+1}\right) \cap \widetilde{C}_{T}\left(B_{p}^{\frac{N}{p}-1}\right) .\right.
$$

2. Uniqueness: If in addition $1 \leq p \leq N$ then uniqueness holds in $F_{p}^{\frac{N}{p}}$.

Moreover we have a control on the time $T$ which may be bounded from below by:

$$
\min \left(\eta, \max \left(t>0, \sum_{q \in \mathbb{Z}} 2^{q\left(\frac{N}{p}-1\right)}\left\|\Delta_{q} u_{0}\right\|_{L^{p}}\left(\frac{1-e^{-c \widetilde{\nu} t e^{2 q}}}{c \widetilde{\nu}}\right) \leq \frac{\varepsilon \widetilde{\nu}^{2}}{\widetilde{\nu}+U_{0}}\right)\right) .
$$

where $\widetilde{\nu}=\min (\mu(\bar{\rho}), \lambda(\bar{\rho})+2 \mu(\bar{\rho}))$ and $U_{0}=\left\|u_{0}\right\|_{B^{\frac{N}{p}}}$.

In the next theorem, we consider the case when the initial variational density $q_{0}$ belongs to $\bar{\rho}+\widetilde{B}_{p}^{\frac{N}{p}, \frac{N}{p}+\varepsilon}$ with $\varepsilon>0$ and satisfies $0<\underline{\rho}<\rho$. Here we do not suppose a smallness condition on $\left\|q_{0}\right\|_{B^{\frac{N}{p}}}$ but we impose more regularity.

Theorem 1.3 Let $\varepsilon \in] 0,1\left[\right.$ and $p \in\left[1, \frac{N}{1-\varepsilon}\right.$ [ and we assume that the physical coefficients verify the same hypothesis as in theorem 1.1. Let $\rho_{0} \in \bar{\rho}+\widetilde{B}_{p}^{\frac{N}{p}, \frac{N}{p}+\varepsilon}$ for a constant $\bar{\rho}>0$, $u_{0} \in B^{\frac{N}{p}+\varepsilon-1}$. Assume that there is a constant $\underline{\rho}$ such that:

$$
0<\underline{\rho} \leq \rho_{0} .
$$

There exists a time $T>0$ such that system $(S W)$ has a unique solution $(q, u)$ in $F_{p+\varepsilon}^{\frac{N}{p}}$. Moreover we have a control on the time $T$ which may be bounded from below by:

$$
\min \left(\eta, \max \left(t>0, \sum_{q \in \mathbb{Z}} 2^{q\left(\frac{N}{p}-1\right)}\left\|\Delta_{q} u_{0}\right\|_{L^{p}}\left(\frac{1-e^{-c \widetilde{\nu} t e^{2 q}}}{c \widetilde{\nu}}\right) \leq \frac{\varepsilon \widetilde{\nu}^{2}}{\widetilde{\nu}+U_{0}}\right)\right) .
$$

where: $\widetilde{\nu}=\min (\mu(\bar{\rho}), \lambda(\bar{\rho})+2 \mu(\bar{\rho}))$ and $U_{0}=\left\|u_{0}\right\|_{B^{\frac{N}{p}}}$.

The present chapter is structured as follows.

In the Section 2, we recall some basic facts about Littlewood-Paley decomposition and Besov spaces.

In the Section 3 we prove the theorem 1.1 and in the Section 4 we show the theorem 1.2 We conclude in the Section 5 by the proof of the theorem 1.3 , 


\section{Littlewood-Paley theory and Besov spaces}

\subsection{Littlewood-Paley decomposition}

Littlewood-Paley decomposition corresponds to a dyadic decomposition of the space in Fourier variables.

We can use for instance any $\varphi \in C^{\infty}\left(\mathbb{R}^{N}\right)$, supported in $\mathcal{C}=\left\{\xi \in \mathbb{R}^{N} / \frac{3}{4} \leq|\xi| \leq \frac{8}{3}\right\}$ such that:

$$
\sum_{l \in \mathbb{Z}} \varphi\left(2^{-l} \xi\right)=1 \text { if } \xi \neq 0
$$

Denoting $h=\mathcal{F}^{-1} \varphi$, we then define the dyadic blocks by:

$$
\Delta_{l} u=\varphi\left(2^{-l} D\right) u=2^{l N} \int_{\mathbb{R}^{N}} h\left(2^{l} y\right) u(x-y) d y \text { and } S_{l} u=\sum_{k \leq l-1} \Delta_{k} u .
$$

Formally, one can write that:

$$
u=\sum_{k \in \mathbb{Z}} \Delta_{k} u
$$

This decomposition is called homogeneous Littlewood-Paley decomposition. Let us observe that the above formal equality does not hold in $\mathcal{S}^{\prime}\left(\mathbb{R}^{N}\right)$ for two reasons:

1. The right hand-side does not necessarily converge in $\mathcal{S}^{\prime}\left(\mathbb{R}^{N}\right)$.

2. Even if it does, the equality is not always true in $\mathcal{S}^{\prime}\left(\mathbb{R}^{N}\right)$ (consider the case of the polynomials).

However, this equality holds true modulo polynomials hence homogeneous Besov spaces will be defined modulo the polynomials, according to [15].

\subsection{Homogeneous Besov spaces and first properties}

Definition 2.3 For $s \in \mathbb{R}, p \in[1,+\infty], q \in[1,+\infty]$, and $u \in \mathcal{S}^{\prime}\left(\mathbb{R}^{N}\right)$ we set:

$$
\|u\|_{B_{p, q}^{s}}=\left(\sum_{l \in \mathbb{Z}}\left(2^{l s}\left\|\Delta_{l} u\right\|_{L^{p}}\right)^{q}\right)^{\frac{1}{q}}
$$

A difficulty due to the choice of homogeneous spaces arises at this point. Indeed, $\|\cdot\|_{B_{p, q}^{s}}$ cannot be a norm on $\left\{u \in \mathcal{S}^{\prime}\left(\mathbb{R}^{N}\right),\|u\|_{B_{p, q}^{s}}<+\infty\right\}$ because $\|u\|_{B_{p, q}^{s}}=0$ means that $u$ is a polynomial. This enforces us to adopt the following definition for homogeneous Besov spaces, see [15].

Definition 2.4 Let $s \in \mathbb{R}, p \in[1,+\infty], q \in[1,+\infty]$.

Denote $m=\left[s-\frac{N}{p}\right]$ if $s-\frac{N}{p} \notin \mathbb{Z}$ or $q>1$ and $m=s-\frac{N}{p}-1$ otherwise.

- If $m<0$, then we define $B_{p, q}^{s}$ as:

$$
B_{p, q}^{s}=\left\{u \in \mathcal{S}^{\prime}\left(\mathbb{R}^{N}\right) /\|u\|_{B_{p, q}^{s}}<\infty \text { and } u=\sum_{l \in \mathbb{Z}} \Delta_{l} u \text { in } \mathcal{S}^{\prime}\left(\mathbb{R}^{N}\right)\right\} .
$$


- If $m \geq 0$, we denote by $\mathcal{P}_{m}\left[\mathbb{R}^{N}\right]$ the set of polynomials of degree less than or equal to $m$ and we set:

$$
B_{p, q}^{s}=\left\{u \in \mathcal{S}^{\prime}\left(\mathbb{R}^{N}\right) / \mathcal{P}_{m}\left[\mathbb{R}^{N}\right] /\|u\|_{B_{p, q}^{s}}<\infty \text { and } u=\sum_{l \in \mathbb{Z}} \Delta_{l} u \text { in } \mathcal{S}^{\prime}\left(\mathbb{R}^{N}\right) \mathcal{P}_{m}\left[\mathbb{R}^{N}\right]\right\} .
$$

The definition of $B_{p, r}^{s}$ does not depend on the choice of the Littlewood-Paley decomposition.

Remark 1 In the sequel, we will use only Besov space $B_{p, q}^{s}$ with $q=1$ and we will denote them by $B_{p}^{s}$ or even by $B^{s}$ if there is no ambiguity on the index $p$.

Let us now state some basic properties for those Besov spaces.

Proposition 2.1 The following properties holds:

1. Density: If $p<+\infty$ and $|s| \leq N / p$, then $C_{0}^{\infty}$ is dense in $B_{p}^{s}$.

2. Derivatives: there exists a constant universal $C$ such that:

$$
C^{-1}\|u\|_{B_{p, r}^{s}} \leq\|\nabla u\|_{B_{p, r}^{s-1}} \leq C\|u\|_{B_{p, r}^{s}} .
$$

3. Sobolev embeddings: If $p_{1}<p_{2}$ and $r_{1} \leq r_{2}$ then $B_{p_{1}, r_{1}}^{s} \hookrightarrow B_{p_{2}, r_{2}}^{s-N\left(\frac{1}{p_{1}}-\frac{1}{p_{2}}\right)}$.

4. Algebraic properties: For $s>0, B_{p, r}^{s} \cap L^{\infty}$ is an algebra. Moreover, for any $p \in[1,+\infty]$ then $B_{p, 1}^{\frac{N}{p}} \hookrightarrow B_{p, \infty}^{\frac{N}{p}} \cap L^{\infty}$, and $B_{p, 1}^{\frac{N}{p}}$ is an algebra if $p$ is finite.

5. Real interpolation: $\left(B_{p, r}^{s_{1}}, B_{p, r}^{s_{2}}\right)_{\theta, r^{\prime}}=B_{p, r^{\prime}}^{\theta s_{1}+(1-\theta) s_{2}}$.

\subsection{Hybrid Besov spaces and Chemin-Lerner spaces}

Hybrid Besov spaces are functional spaces where regularity assumptions are different in low frequency and high frequency, see [15]. We are going to give the definition of this this new spaces and give some of their main properties.

Definition 2.5 Let $s, t \in \mathbb{R}$. We set:

$$
\|u\|_{\widetilde{B}_{p, r}^{s, t}}=\left(\sum_{q \leq 0}\left(2^{q s}\left\|\Delta_{q} u\right\|_{L^{p}}\right)^{r}\right)^{\frac{1}{r}}+\left(\sum_{q>0}\left(2^{q t}\left\|\Delta_{q} u\right\|_{L^{p}}\right)^{r}\right)^{\frac{1}{r}} .
$$

Denote $m=\left[s-\frac{N}{p}\right]$ if $s-\frac{N}{p} \notin \mathbb{Z}$ or $r>1$ and $m=s-\frac{N}{p}-1$ otherwise, we then define:

- $\widetilde{B}_{p}^{s, t}=\left\{u \in \mathcal{S}^{\prime}\left(\mathbb{R}^{N}\right) /\|u\|_{\widetilde{B}_{p}^{s, t}}<+\infty\right\}$, if $m<0$

- $\widetilde{B}_{p}^{s, t}=\left\{u \in \mathcal{S}^{\prime}\left(\mathbb{R}^{N}\right) / \mathcal{P}_{m}\left[\mathbb{R}^{N}\right] /\|u\|_{\widetilde{B}_{p}^{s, t}}<+\infty\right\}$ if $m \geq 0$.

Let now give some properties of these hybrid spaces and some results on how they behave with respect to the product. The following results come directly from the paradifferential calculus. 
Proposition 2.2 We give here some results of inclusion:

1. We have $\widetilde{B}_{p, r}^{s, s}=B_{p, r}^{s}$.

2. If $s \leq t$ then $\widetilde{B}_{p, r}^{s, t}=B_{p, r}^{s} \cap B_{p, r}^{t}$ or if $s>t$ then $\widetilde{B}_{p, r}^{s, t}=B_{p, r}^{s}+B_{p, r}^{t}$.

3. If $s_{1} \leq s_{2}$ and $t_{1} \geq t_{2}$ then $\widetilde{B}_{p, r}^{s_{1}, t_{1}} \hookrightarrow \widetilde{B}_{p, r}^{s_{2}, t_{2}}$.

Proposition 2.3 For all $s, t>0,1 \leq r, p \leq+\infty$, the following inequality holds true:

$$
\|u v\|_{\widetilde{B}_{p, r}^{s, t}} \leq C\left(\|u\|_{L^{\infty}}\|v\|_{\widetilde{B}_{p, r}^{s, t}}+\|v\|_{L^{\infty}}\|u\|_{\widetilde{B}_{P, r}^{s, t}}\right) .
$$

For all $s_{1}, s_{2}, t_{1}, t_{2} \leq \frac{N}{p}$ such that $\min \left(s_{1}+s_{2}, t_{1}+t_{2}\right)>0$ we have:

$$
\begin{gathered}
\|u v\|_{\widetilde{B}_{p, r}^{s_{1}+t_{1}-\frac{N}{p}, s_{2}+t_{2}-\frac{N}{p}}} \leq C\|u\|_{\widetilde{B}_{p, r}^{s_{1}, t_{1}}}\|v\|_{\widetilde{B}_{p, \infty}^{s_{2}, t_{2}}} . \\
\|u v\|_{B_{p, r}^{s}} \leq C\|u\|_{B_{p, r}^{s}}\|v\|_{B_{p, \infty}^{\frac{N}{p}} \cap L^{\infty}} i f|s|<\frac{N}{p} .
\end{gathered}
$$

For a proof of this proposition see [15]. The limit case $s_{1}+s_{2}=t_{1}+t_{2}=0$ in (2.3) is of interest. When $p \geq 2$, the following estimate holds true whenever $s$ is in the range $\left(-\frac{N}{p}, \frac{N}{p}\right]$ (see e.g. [37]):

$$
\|u v\|_{B_{p, \infty}^{-\frac{N}{p}}} \leq C\|u\|_{B_{p, 1}^{s}}\|v\|_{B_{p, \infty}^{-s}} .
$$

The study of non stationary PDE's requires space of type $L^{\rho}(0, T, X)$ for appropriate Banach spaces $X$. In our case, we expect $X$ to be a Besov space, so that it is natural to localize the equation through Littlewood-Payley decomposition. But, in doing so, we obtain bounds in spaces which are not type $L^{\rho}(0, T, X)$ (except if $r=p$ ). We are now going to define the spaces of Chemin-Lerner in which we will work (see [8]), which are a refinement of the spaces $L_{T}^{\rho}\left(B_{p, r}^{s}\right)$.

Definition 2.6 Let $\rho \in[1,+\infty], T \in[1,+\infty]$ and $s_{1}, s_{2} \in \mathbb{R}$. We then denote:

$$
\|u\|_{\widetilde{L}_{T}^{\rho}\left(\widetilde{B}_{p, r}^{s_{1}, s_{2}}\right)}=\left(\sum_{l \leq 0} 2^{l r s_{1}}\left(\left\|\Delta_{l} u(t)\right\|_{L_{T}^{\rho}\left(L^{p}\right)}^{r}\right)^{\frac{1}{r}}+\left(\sum_{l>0} 2^{l r s_{2}}\left(\int_{0}^{T}\left\|\Delta_{l} u(t)\right\|_{L^{p}}^{\rho} d t\right)^{\frac{r}{\rho}}\right)\right)^{\frac{1}{r}} .
$$

We note that thanks to Minkowsky inequality we have:

$$
\begin{array}{ll}
\|u\|_{L_{T}^{\rho}\left(\widetilde{B}_{p, r}^{s_{1}, s_{2}}\right)} \leq\|u\|_{\widetilde{L}_{T}^{\rho}\left(\widetilde{B}_{p, r}^{s_{1}, s_{2}}\right)} & \text { if } \rho \leq r, \\
\|u\|_{\widetilde{L}_{T}^{\rho}\left(\widetilde{B}_{p, r}^{s_{1}, s_{2}}\right)} \leq\|u\|_{L_{T}^{\rho}\left(\widetilde{B}_{p, r}^{s_{1}, s_{2}}\right)} & \text { if } \rho \geq r .
\end{array}
$$

We then define the space:

$$
\widetilde{L}_{T}^{\rho}\left(\widetilde{B}_{p}^{s_{1}, s_{2}}\right)=\left\{u \in L_{T}^{\rho}\left(\widetilde{B}_{p}^{s_{1}, s_{2}}\right) /\|u\|_{\widetilde{L}_{T}^{\rho}\left(\widetilde{B}_{p}^{s_{1}, s_{2}}\right)}<\infty\right\} .
$$

We denote moreover by $\widetilde{C}_{T}\left(\widetilde{B}_{p}^{s_{1}, s_{2}}\right)$ the set of those functions of $\widetilde{L}_{T}^{\infty}\left(\widetilde{B}_{p}^{s_{1}, s_{2}}\right)$ which are continuous from $[0, T]$ to $\widetilde{B}_{p}^{s_{1}, s_{2}}$. In the sequel we are going to give some properties of this spaces concerning the interpolation and their relationship with the heat equation. 
Proposition 2.4 Let $s, t, s_{1}, s_{2} \in \mathbb{R}, r, \rho, \rho_{1}, \rho_{2} \in[1,+\infty]$. We have:

1. Interpolation:

$$
\|u\|_{\tilde{L}_{T}^{\rho}\left(\tilde{B}_{p, r}^{s, t}\right)} \leq\|u\|_{\tilde{L}_{T}^{\rho_{1}}\left(\tilde{B}_{p, r}^{s_{1}, t_{1}}\right)}^{\theta}\|u\|_{\tilde{L}_{T}^{\rho_{2}\left(\tilde{B}_{p, r}^{s_{2}, t_{2}}\right)}}^{1-\theta}
$$

with $\frac{1}{\rho}=\frac{\theta}{\rho_{1}}+\frac{1-\theta}{\rho_{2}}, s=\theta s_{1}+(1-\theta) s_{2}$ and $t=\theta t_{1}+(1-\theta) t_{2}$.

2. Embedding:

$$
\tilde{L}_{T}^{\rho}\left(\tilde{B}_{p}^{s, t}\right) \hookrightarrow L_{T}^{\rho}\left(C_{0}\right) \text { and } \tilde{C}_{T}\left(B_{p}^{\frac{N}{p}}\right) \hookrightarrow C\left([0, T] \times \mathbb{R}^{d}\right)
$$

Here we recall a result of interpolation which explains the link of the space $B_{p, 1}^{s}$ with the homogeneous spaces, see [14].

Proposition 2.5 There exists a constant $C$ such that for all $s \in \mathbb{R}, \varepsilon>0$ and $1 \leq p \leq$ $+\infty$, we have

$$
\|u\|_{B_{p, 1}^{s}} \leq C \frac{1+\varepsilon}{\varepsilon}\|u\|_{B_{p, \infty}^{s}}\left(1+\log \frac{\|u\|_{B_{p, \infty}^{s-\varepsilon}}+\|u\|_{B_{p, \infty}^{s+\varepsilon}}}{\|u\|_{B_{p, \infty}^{s}}}\right) .
$$

To finish with we adapt the results of the paradifferential calculus on the product of Besov function to the spaces of Chemin-Lerner. So we have the following properties:

Proposition 2.6 Let $p, r \in[1,+\infty]$. We have the two following properties:

- Let $s>0, t>0,1 / \rho_{2}+1 / \rho_{3}=1 / \rho_{1}+1 / \rho_{4}=1 / \rho \leq 1, u \in \tilde{L}_{T}^{\rho_{3}}\left(\tilde{B}_{p, r}^{s, t}\right) \cap \tilde{L}_{T}^{\rho_{1}}\left(L^{\infty}\right)$ and $v \in \tilde{L}_{T}^{\rho_{4}}\left(\tilde{B}_{p, r}^{s, t}\right) \cap \tilde{L}_{T}^{\rho_{2}}\left(L^{\infty}\right)$. Then $u v \in \tilde{L}_{T}^{\rho}\left(\tilde{B}_{p, r}^{s, t}\right)$ and we have:

$$
\|u v\|_{\tilde{L}_{T}^{\rho}\left(\tilde{B}_{p, r}^{s, t}\right)} \lesssim\|u\|_{\tilde{L}_{T}^{\rho_{1}}\left(L^{\infty}\right)}\|v\|_{\tilde{L}_{T}^{\rho_{4}}\left(\tilde{B}_{p, r}^{s, t}\right)}+\|v\|_{\tilde{L}_{T}^{\rho_{2}}\left(L^{\infty}\right)}\|u\|_{\tilde{L}_{T}^{\rho_{3}}\left(\tilde{B}_{p, r}^{s, t}\right)}
$$

- If $s_{1}, s_{2}, t_{1}, t_{2} \leq \frac{N}{p}, s_{1}+s_{2}>0, t_{1}+t_{2}>01 / \rho_{1}+1 / \rho_{2}=1 / \rho \leq 1, u \in \tilde{L}_{T}^{\rho_{1}}\left(B_{p, r}^{s_{1}, t_{1}}\right)$ and $v \in \tilde{L}_{T}^{\rho_{2}}\left(B_{p, r}^{s_{2}, t_{2}}\right)$ then $u v \in \tilde{L}_{T}^{\rho}\left(B_{2}^{s_{1}+s_{2}-d / 2}\right)$ and

$$
\|u v\|_{\tilde{L}_{T}^{\rho}\left(B_{p, r}^{\left.s_{1}+s_{2}-\frac{N}{p}, t_{1}+t_{2}-\frac{N}{p}\right)}\right.} \lesssim\|u\|_{\tilde{L}_{T}^{\rho_{1}\left(B_{p, r}^{s_{1}, t_{1}}\right)}}\|v\|_{\tilde{L}_{T}^{\rho_{2}}\left(B_{p, r}^{s_{2}, t_{2}}\right)} .
$$

The analogous of the endpoint estimate (2.5) reads (for $p \geq 2$ ):

$$
\|u v\|_{\widetilde{L}_{T}^{\rho}\left(B_{p, \infty}^{\left.-\frac{N}{p}\right)}\right.} \lesssim\|u\|_{\widetilde{L}_{T}^{\rho_{1}}\left(B_{p, 1}^{s}\right)}\|u\|_{\widetilde{L}_{T}^{\rho_{2}\left(B_{p, \infty}^{-s}\right)}},
$$

whenever $s$ is in the range $\left(-\frac{N}{p}, N N\right]$ and $\frac{1}{\rho_{1}}+\frac{1}{\rho_{2}}=\frac{1}{\rho} \leq 1$ (see the proof in [19]). For a proof of this proposition see [15]. Finally we need an estimate on the composition of functions in the spaces $\tilde{L}_{T}^{\rho}\left(\tilde{B}_{p}^{s}\right)$. 
Proposition 2.7 Let $s>0, r \in[1,+\infty]$ and $F \in W_{l o c}^{s+2, \infty}\left(\mathbb{R}^{N}\right)$ such that $F(0)=0$. There exists a function $C$ depending only on $s, p, N$ and $F$, and such that:

$$
\|F(u)\|_{\tilde{L}_{T}^{\rho}\left(\tilde{B}_{p, r}^{s_{1}, s_{2}}\right)} \leq C\left(\|u\|_{L_{T}^{\infty}\left(L^{\infty}\right)}\right)\|u\|_{\tilde{L}_{T}^{\rho}\left(\tilde{B}_{p, r}^{s_{1}, s_{2}}\right)} .
$$

If $v, u \in \tilde{L}_{T}^{\rho}\left(B_{p}^{s_{1}, s_{2}}\right) \cap L_{T}^{\infty}\left(L^{\infty}\right)$ and $G \in W_{l o c}^{[s]+3, \infty}\left(\mathbb{R}^{N}\right)$ then $G(u)-G(v)$ belongs to $\tilde{L}_{T}^{\rho}\left(B_{p}^{s_{1}, s_{2}}\right)$ and it exists a constant $C$ depending only of $s, p, N$ and $G$ such that:

$$
\begin{aligned}
& \|G(u)-G(v)\|_{\tilde{L}_{T}^{\rho}\left(B_{p, r}^{s_{1}, s_{2}}\right)} \leq C\left(\|u\|_{L_{T}^{\infty}\left(L^{\infty}\right)},\|v\|_{L_{T}^{\infty}\left(L^{\infty}\right)}\right)\left(\|v-u\|_{\tilde{L}_{T}^{\rho}\left(B_{p, r}^{s_{1}, s_{2}}\right)}\right. \\
& \quad\left(1+\|u\|_{L_{T}^{\infty}\left(L^{\infty}\right)}+\|v\|_{L_{T}^{\infty}\left(L^{\infty}\right)}\right)+\|v-u\|_{L_{T}^{\infty}\left(L^{\infty}\right)}\left(\|u\|_{\tilde{L}_{T}^{\rho}\left(B_{p, r}^{s_{1}, s_{2}}\right)}+\|v\|_{\tilde{L}_{T}^{\rho}\left(B_{p, r}^{s_{1}, s_{2}}\right)}\right) .
\end{aligned}
$$

The proof is a adaptation of a theorem by J.Y. Chemin and H. Bahouri in [1].

We end this section by recalling some estimates in Besov spaces for transport and heat equations. For more details, the reader is referred to [8] and [17].

Proposition 2.8 Let $(p, r) \in[1,+\infty]^{2}$ and $s \in\left(-\min \left(\frac{N}{p}, \frac{N}{p^{T}}\right), \frac{N}{p}+1\right)$. Let $u$ be a vector field such that $\nabla u$ belongs to $L^{1}\left(0, T ; B_{p, r}^{\frac{N}{p}} \cap L^{\infty}\right)$. Suppose that $q_{0} \in B_{p, r}^{s}, F \in$ $L^{1}\left(0, T, B_{p, r}^{s}\right)$ and that $q \in L_{T}^{\infty}\left(B_{p, r}^{s}\right) \cap C\left([0, T] ; \mathcal{S}^{\prime}\right)$ solves the following transport equation:

$$
\left\{\begin{array}{l}
\partial_{t} q+u \cdot \nabla q=F, \\
q_{t=0}=q_{0} .
\end{array}\right.
$$

Let $U(t)=\int_{0}^{t}\|\nabla u(\tau)\|_{B_{p}^{\frac{N}{p}} \cap L^{\infty}} d \tau$. There exits a constant $C$ depending only on $s, p$ and $N$, and such that for all $t \in[0, T]$, the following inequality holds:

$$
\|q\|_{\widetilde{L}_{t}^{\infty}\left(B_{p, r}^{s}\right)} \leq \exp ^{C U(t)}\left(\left\|q_{0}\right\|_{B_{p, r}^{s}}+\int_{0}^{t} \exp ^{-C U(\tau)}\|F(\tau)\|_{B_{p, r}^{s}} d \tau\right)
$$

If $r<+\infty$ then $q$ belongs to $C\left([0, T] ; B_{p, r}^{s}\right)$.

Actually, in [17, the proposition below is proved for non-homogeneous Besov spaces. The adaptation to homogeneous spaces is straightforward. Let us now some estimates for the heat equation:

Proposition 2.9 Let $s \in \mathbb{R},(p, r) \in[1,+\infty]^{2}$ and $1 \leq \rho_{2} \leq \rho_{1} \leq+\infty$. Assume that $u_{0} \in B_{p, r}^{s}$ and $f \in \widetilde{L}_{T}^{\rho_{2}}\left(\widetilde{B}_{p, r}^{s-2+2 / \rho_{2}}\right)$. Let $u$ be a solution of:

$$
\left\{\begin{array}{l}
\partial_{t} u-\mu \Delta u=f \\
u_{t=0}=u_{0} .
\end{array}\right.
$$

Then there exists $C>0$ depending only on $N, \mu, \rho_{1}$ and $\rho_{2}$ such that:

$$
\|u\|_{\widetilde{L}_{T}^{\rho_{1}}\left(\widetilde{B}_{p, r}^{s+2 / \rho_{1}}\right)} \leq C\left(\left\|u_{0}\right\|_{B_{p, r}^{s}}+\mu^{\frac{1}{\rho_{2}}-1}\|f\|_{\widetilde{L}_{T}^{\rho_{2}}\left(\widetilde{B}_{p}^{s-2+2 / \rho_{2}}\right)}\right) .
$$

If in addition $r$ is finite then $u$ belongs to $C\left([0, T], B_{p, r}^{s}\right)$. 
The proof of local well-posedness for initial density bounded away from zero requires estimates in $B_{p}^{s}$ spaces for the following linear system:

$$
\left\{\begin{array}{l}
\partial_{t} u-\bar{\mu} \operatorname{div}(a \nabla u)-(\bar{\lambda}+\bar{\mu}) \nabla(a \operatorname{div} u)=G, \\
u_{t=0}=u_{0},
\end{array}\right.
$$

where $u(t, x) \in \mathbb{R}^{N}, \bar{\nu}=2 \bar{\mu}+\bar{\lambda}>0$ and the diffusion coefficient is assumed to satisfy:

$$
0<\underline{a} \leq a(t, x) \leq \bar{a} .
$$

We can prove that the solution of the previous system satisfy estimates analogous to those of proposition [2.9, see [15].

Proposition 2.10 Let $1<p<+\infty, 1 \leq \alpha_{1} \leq r \leq+\infty$ and $s$ be such that $\max \left(1, \frac{N}{p}\right) \leq$ $s \leq \frac{N}{p}+1$. Set $\alpha_{2}^{\prime}=\frac{2}{s-\frac{N}{p}}$ and let $\alpha_{2}$ be such that $\frac{1}{\alpha_{1}}=\frac{1}{\alpha_{2}}+\frac{1}{\alpha_{2}^{\prime}}$. Let assumption (2.7) be fulfilled and $u$ be a solution of (2.3). We suppose that the regularity index $\tau$ satisfies:

$$
1-\frac{2}{\alpha_{1}}-\frac{N}{p}<\tau \leq 1-\frac{2}{\alpha_{1}}+s .
$$

Then the following estimate holds for all $\alpha \in[r,+\infty]$ :

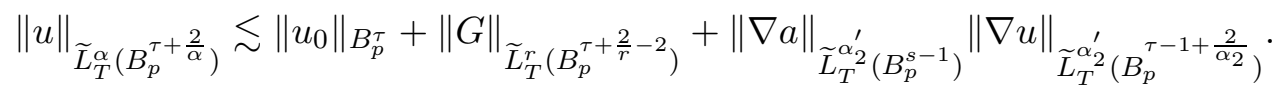

\section{Proof of theorem 1.1}

\subsection{Sketch of the Proof}

In this section, we give the sketch of the proof of theorem 1.1 on the global existence result with small initial data.

We will suppose that $\rho$ is close to a constant state $\bar{\rho}$, so that $\rho$ will be strictly superior to a positive constant, we will use the parabolicity of the momentum equation to get a gain of derivatives on the velocity $u$. The density $\rho$ has a behavior similar to the solution of a transport equation. Let us rewrite the $(S W)$ system in a non conservative form in using the definition 1.2 .

$$
\left\{\begin{aligned}
& \partial_{t} q+u \cdot \nabla q+\operatorname{div} u=F \\
& \begin{array}{rl}
\partial_{t} u+u \cdot \nabla u-\frac{\mu(\bar{\rho})}{\bar{\rho}} \Delta u-\frac{\mu(\bar{\rho})+\lambda(\bar{\rho})}{\bar{\rho}} \nabla \operatorname{div} u+\left(\kappa \bar{\rho}+P^{\prime}(\bar{\rho})\right) & \nabla q \\
& -\kappa \bar{\rho} \phi * \nabla q=G
\end{array}
\end{aligned}\right.
$$

where we have:

$$
\begin{aligned}
& F=-q \operatorname{div} u, \\
& G=\mathcal{A}(\rho, u)+K(\rho) \nabla q,
\end{aligned}
$$


where we set:

$$
\begin{aligned}
& \mathcal{A}(\rho, u)=\left[\frac{\operatorname{div}(\mu(\rho) D(u))}{\rho}-\frac{\mu(\bar{\rho})}{\bar{\rho}} \Delta u\right]+\left[\frac{\nabla((\mu(\rho)+\lambda(\rho)) \operatorname{div} u)}{\rho}-\frac{\mu(\bar{\rho})+\lambda(\bar{\rho})}{\bar{\rho}} \nabla \operatorname{div} u\right], \\
& K(\rho)=\frac{\bar{\rho} P^{\prime}(\rho)}{\rho}-P^{\prime}(\bar{\rho}) .
\end{aligned}
$$

For $s \in \mathbb{R}$, we denote $\Lambda^{s} h=\mathcal{F}^{-1}\left(|\xi|^{s} \widehat{h}\right)$. We set now:

$$
d=\Lambda^{-1} \operatorname{div} u \text { and } \Omega=\Lambda^{-1} \operatorname{curl} u
$$

where $d$ represents the compressible part of the velocity and $\Omega$ the incompressible part. We rewrite now the system $(S W 1)$ in using these previous notations on a linear form:

$$
\left\{\begin{array}{l}
\partial_{t} q+\Lambda d=F_{1} \\
\partial_{t} d-\bar{\nu} \Delta d-\bar{\delta} \Lambda q+\bar{\kappa} \Lambda(\phi * q)=G_{1} \\
\partial_{t} \Omega-\bar{\mu} \Delta \Omega=H_{1} \\
u=-\Lambda^{-1} \nabla d-\Lambda \operatorname{div} \Omega
\end{array}\right.
$$

where we have :

$$
\bar{\mu}=\frac{\mu(\bar{\rho})}{\bar{\rho}}, \quad \bar{\lambda}=\frac{\lambda(\bar{\rho})}{\bar{\rho}}, \quad \bar{\nu}=2 \bar{\mu}+\bar{\lambda}, \quad \bar{\delta}=\kappa \bar{\rho}+P^{\prime}(\bar{\rho}), \quad \text { and } \quad \bar{\kappa}=\kappa \bar{\rho} .
$$

We have in our case:

$$
\begin{aligned}
& F_{1}=-q \operatorname{div} u-u \cdot \nabla q, \\
& G_{1}=-\Lambda^{-1} \operatorname{div}(G), \\
& H_{1}=-\Lambda^{-1} \operatorname{curl}(G) .
\end{aligned}
$$

The first idea will be to study the linear system associated to $(S W 2)$. We concentrate on the first two equations because the third equation is just a heat equation with a non linear term. The system we want to study reads:

$$
\left\{\begin{array}{l}
\partial_{t} q+\Lambda d=F^{\prime} \\
\partial_{t} d-\bar{\nu} \Delta d-\bar{\delta} \Lambda q+\bar{\kappa} \Lambda(\phi * q)=G^{\prime} .
\end{array}\right.
$$

This system has been studied by D. Hoff and K. Zumbrum in [28] in the case $\bar{\kappa}=0$. There, they investigate the decay estimates, and exhibit the parabolic smoothing effect on $d$ and on the low frequencies of $q$, and a damping effect on the high frequencies of $q$. The problem is that if we focus on this linear system, it appears impossible to control the term of convection $u \cdot \nabla q$ which is one derivative less regular than $q$. Hence we shall include the convection term in the linear system. We thus have to study:

$$
\left\{\begin{array}{l}
\partial_{t} q+v \cdot \nabla q+\Lambda d=F \\
\partial_{t} d+v \cdot \nabla d-\bar{\nu} \Delta d-\bar{\delta} \Lambda q+\bar{\kappa} \Lambda(\phi * q)=G,
\end{array}\right.
$$

where $v$ is a function and we will precise its regularity in the next proposition. System $(S W 2)^{\prime}$ has been studied in the case where $\phi=0$ by R. Danchin in [15], we then adapt the proof in taking into consideration the term coming from the capillarity.

In the sequel we will assume $\bar{\nu}>0$ and $\bar{\delta}-\bar{\kappa}\|\widehat{\phi}\|_{L^{\infty}} \geq c>0$. We get then the following proposition. 
Proposition 3.11 Let $(q, d)$ a solution of the system $(S W 2)^{\prime}$ on $\left[0, T\left[, 1-\frac{N}{2}<s \leq\right.\right.$ $1+\frac{N}{2}$ and $V(t)=\int_{0}^{t}\|v(\tau)\|_{B^{\frac{N}{2}+1}} d \tau$. We have then the following estimate:

$$
\begin{aligned}
& \|(q, d)\|_{\widetilde{B}^{s-1, s} \times B^{s-1}}+\int_{0}^{t}\|(q, d)(\tau)\|_{\widetilde{B}^{s+1, s} \times B^{s+1}} d \tau \\
& \leq C e^{C V(t)}\left(\left\|\left(q_{0}, d_{0}\right)\right\|_{\widetilde{B}^{s-1, s} \times B^{s-1}}+\int_{0}^{t} e^{-C V(\tau)}\|(F, G)(\tau)\|_{\widetilde{B}^{s-1, s} \times B^{s-1}} d \tau\right),
\end{aligned}
$$

where $C$ depends only on $\bar{\nu}, \bar{\delta}, \bar{\kappa}, \phi, N$ and $s$.

\section{Proof of Proposition 3.11;}

Let $(q, u)$ be a solution of $(S W 2)^{\prime}$ and we set:

$$
\widetilde{q}=e^{-K V(t)} q, \widetilde{u}=e^{-K V(t)} u, \widetilde{F}=e^{-K V(t)} F \text { and } \widetilde{G}=e^{-K V(t)} G .
$$

We are going to separate the case of the low and high frequencies, which have a different behavior concerning the control of the derivative index for the Besov spaces.

In this goal we will consider the two different expressions in low and high frequencies where $l_{0} \in \mathbb{Z}, A, B$ and $K_{1}$ will be fixed later in the proof:

$$
\begin{aligned}
& f_{l}^{2}=\bar{\delta}\left\|\widetilde{q}_{l}\right\|_{L^{2}}^{2}-\bar{\kappa}\left(\widetilde{q}_{l}, \phi * \widetilde{q}_{l}\right)+\left\|\widetilde{d}_{l}\right\|_{L^{2}}^{2}-2 K_{1}\left(\Lambda \widetilde{q}_{l}, \widetilde{d}_{l}\right) \quad \text { for } l \leq l_{0}, \\
& f_{l}^{2}=\left\|\Lambda \widetilde{q}_{l}\right\|_{L^{2}}^{2}+A\left\|\widetilde{d}_{l}\right\|_{L^{2}}^{2}-\frac{2}{\bar{\nu}}\left(\Lambda \widetilde{q}_{l}, \widetilde{d}_{l}\right) \quad \text { for } l>l_{0} .
\end{aligned}
$$

In the first two steps, we show that $K_{1}$ and $A$ may be chosen such that:

$$
2^{l(s-1)} f_{l}^{2} \approx 2^{l s} \max \left(1,2^{-l}\right)\left\|\widetilde{q}_{l}\right\|_{L^{2}}^{2}+2^{l(s-1)}\left\|\widetilde{d}_{l}\right\|_{L^{2}}^{2},
$$

and we will show the following inequality:

$$
\begin{aligned}
\frac{1}{2} \frac{d}{d t} f_{l}^{2}+\alpha \min \left(2^{2 l}, 1\right) f_{l}^{2} \leq C 2^{-l(s-1)} \alpha_{l} f_{l} & \left(\|(\widetilde{F}, \widetilde{G})\|_{\widetilde{B}^{s-1, s} \times B^{s-1}}\right. \\
& \left.+V^{\prime}\|(\widetilde{q}, \widetilde{d})\|_{\widetilde{B}^{s-1, s} \times B^{s-1}}\right)-K V^{\prime} f_{l}^{2}
\end{aligned}
$$

where $\sum_{l \in \mathbb{Z}} \alpha_{l} \leq 1$ and $\alpha$ is a positive constant.

This inequality enables us to get a decay for $q$ and $d$ which will be used to show a smoothing parabolic effect on $d$.

\section{Case of low frequencies}

Applying operator $\Delta_{l}$ to the system $(S W 2)^{\prime}$, we obtain then in setting:

$$
\widetilde{q}_{l}=\Delta_{l} \widetilde{q}, \widetilde{d}_{l}=\Delta_{l} \widetilde{d} .
$$

the following system:

$$
\left\{\begin{array}{l}
\frac{d}{d t} \widetilde{q}_{l}+\Delta_{l}(v \cdot \nabla \widetilde{q})+\Lambda \widetilde{d}_{l}=\widetilde{F}_{l}-K V^{\prime}(t) \widetilde{q}_{l}, \\
\frac{d}{d t} d_{l}+\Delta_{l}\left(v \cdot \nabla \widetilde{d}_{l}\right)-\bar{\nu} \Delta \widetilde{d}_{l}-\bar{\delta} \Lambda \widetilde{q}_{l}+\bar{\kappa} \Lambda\left(\phi * \widetilde{q}_{l}\right)=\widetilde{G}_{l}-K V^{\prime}(t) \widetilde{d}_{l} .
\end{array}\right.
$$


We set:

$$
f_{l}^{2}=\bar{\delta}\left\|\widetilde{q}_{l}\right\|_{L^{2}}^{2}+\left\|\widetilde{d}_{l}\right\|_{L^{2}}^{2}-2 K_{1}\left(\Lambda \widetilde{q}_{l}, \widetilde{d}_{l}\right)
$$

for some $K_{1} \geq 0$ to be fixed hereafter and $(\cdot, \cdot)$ noting the $L^{2}$ inner product.

To begin with, we consider the case where $F=G=0, v=0$ and $K=0$. Taking the $L^{2}$ scalar product of the first equation of (3.12) with $\tilde{q}_{l}$ and of the second equation with $\tilde{d}_{l}$, we get the following two identities:

$$
\left\{\begin{array}{l}
\frac{1}{2} \frac{d}{d t}\left\|q_{l}\right\|_{L^{2}}^{2}+\left(\Lambda d_{l}, q_{l}\right)=0 \\
\frac{1}{2} \frac{d}{d t}\left\|d_{l}\right\|_{L^{2}}^{2}+\bar{\nu}\left\|\Lambda d_{l}\right\|_{L^{2}}^{2}-\bar{\delta}\left(\Lambda q_{l}, d_{l}\right)+\bar{\kappa}\left(\Lambda\left(\phi * q_{l}\right), d_{l}\right)=0 .
\end{array}\right.
$$

In the same way we have:

$$
\frac{1}{2} \frac{d}{d t}\left(q_{l}, q_{l} * \phi\right)+\left(\Lambda d_{l}, \phi * q_{l}\right)=0
$$

because we have by the theorem of Plancherel:

$$
\left(\frac{d}{d t} q_{l}, q_{l} * \phi\right)=\left(\frac{d}{d t} \widehat{q_{l}}, \widehat{q_{l}} \widehat{\phi}\right)=\frac{1}{2} \frac{d}{d t}\left(\widehat{q_{l}}, \widehat{q_{l}} \widehat{\phi}\right)=\frac{1}{2} \frac{d}{d t}\left(q_{l}, q_{l} * \phi\right) .
$$

We want now get an equality involving $\bar{\nu}\left(\Lambda d_{l}, q_{l}\right)$. To achieve it, we apply $\bar{\nu} \Lambda$ to the first equation of (3.12) and take the $L^{2}$-scalar product with $d_{l}$, then take the scalar product of the second equation with $\Lambda q_{l}$ and sum both equalities, which yields:

$$
\frac{d}{d t}\left(\Lambda q_{l}, d_{l}\right)+\left\|\Lambda d_{l}\right\|_{L^{2}}^{2}-\bar{\delta}\left\|\Lambda q_{l}\right\|_{L^{2}}^{2}+\bar{\kappa}\left\|\phi * \Lambda q_{l}\right\|_{L^{2}}^{2}+\bar{\nu}\left(\Lambda^{2} d_{l}, \Lambda q_{l}\right)=0 .
$$

By linear combination of (3.14) and (3.16), we get:

$$
\frac{1}{2} \frac{d}{d t} f_{l}^{2}+\left(\bar{\nu}-K_{1}\right)\left\|\Lambda d_{l}\right\|_{L^{2}}^{2}+K_{1}\left(\bar{\delta}\left\|\Lambda q_{l}\right\|_{L^{2}}^{2}-\bar{\kappa}\left\|\phi * \Lambda q_{l}\right\|_{L^{2}}^{2}\right)-\bar{\nu} K_{1}\left(\Lambda^{2} d_{l}, \Lambda q_{l}\right)=0 .
$$

And as we have assumed that: $\delta-\bar{\kappa}\|\widehat{\phi}\|_{L^{\infty}} \geq c>0$ we get:

$$
\frac{1}{2} \frac{d}{d t} f_{l}^{2}+\left(\bar{\nu}-K_{1}\right)\left\|\Lambda d_{l}\right\|_{L^{2}}^{2}+K_{1} c\left\|q_{l}\right\|_{L^{2}}^{2}-\bar{\nu} K_{1}\left(\Lambda^{2} d_{l}, \Lambda q_{l}\right) \leq 0 .
$$

Using spectral localization for $d_{l}$ and convex inequalities, we find for every $a>0$ :

$$
\left|\left(\Lambda^{2} d_{l}, \Lambda q_{l}\right)\right| \leq \frac{a 2^{2 l_{0}}}{2}\left\|\Lambda d_{l}\right\|_{L^{2}}^{2}+\frac{1}{2 a}\left\|\Lambda q_{l}\right\|_{L^{2}}^{2} .
$$

In using the previous inequality and (3.17), we get:

$$
\frac{1}{2} \frac{d}{d t} f_{l}^{2}+\left(\bar{\nu}-K_{1}-\frac{a 2^{2 l_{0}}}{2}\right)\left\|\Lambda d_{l}\right\|_{L^{2}}^{2}+\left(K_{1} c-\frac{1}{2 a}\right)\left\|\Lambda q_{l}\right\|_{L^{2}}^{2} \leq 0 .
$$

From (3.13) and (3.19) we get in choosing $a=\bar{\nu}$ and $K_{1}<\min \left(\frac{1}{2^{2 l_{0}}}, \frac{\bar{\nu}}{2+2^{2 l_{0}} \bar{\nu}^{2}}\right)$, then:

$$
\frac{1}{2} \frac{d}{d t} f_{l}^{2}+\alpha 2^{2 l} f_{l}^{2} \leq 0
$$


for a constant $\alpha$ depending only on $\bar{\nu}$ and $K_{1}$.

In the general case where $F, G, K$ and $v$ are not zero, we have:

$$
\begin{aligned}
\frac{1}{2} \frac{d}{d t} f_{l}^{2}+\left(\alpha 2^{2 l}+K V^{\prime}\right) f_{l}^{2} \leq & \left(\widetilde{F}_{l}, \widetilde{q}_{l}\right)+\left(\widetilde{G}_{l}, \widetilde{d}_{l}\right)-K\left(\Lambda \widetilde{F}_{l}, \widetilde{d}_{l}\right)-K\left(\Lambda \widetilde{G}_{l}, \widetilde{q}_{l}\right)-\left(\Delta_{l}(v \cdot \nabla \widetilde{q}), \widetilde{q}_{l}\right) \\
& -\left(\Delta_{l}(v \cdot \nabla \widetilde{d}), \widetilde{d}_{l}\right)+K\left(\left(\Lambda \Delta_{l}(v \cdot \nabla \widetilde{q}), \widetilde{d}_{l}\right)+\left(\left(\Lambda \Delta_{l}(v \cdot \nabla \widetilde{d}), \widetilde{q}_{l}\right) .\right.\right.
\end{aligned}
$$

Now we can use a lemma of harmonic analysis in [15] to estimate the last terms, and get the existence of a sequence $\left(\alpha_{l}\right)_{l \in \mathbb{Z}}$ such that $\sum_{l \in \mathbb{Z}} \alpha_{l} \leq 1$ and:

$$
\frac{1}{2} \frac{d}{d t} f_{l}^{2}+\left(\alpha 2^{2 l}+K V^{\prime}\right) f_{l}^{2} \lesssim \alpha_{l} f_{l} 2^{-l(s-1)}\left(\|(\widetilde{F}, \widetilde{G})\|_{\widetilde{B}^{s-1, s} \times B^{s-1}}+V^{\prime}\|(\widetilde{q}, \widetilde{d})\|_{\widetilde{B}^{s-1, s} \times B^{s-1}}\right) .
$$

\section{Case of high frequencies}

We consider now the case where $l \geq l_{0}+1$ and we recall that:

$$
f_{l}^{2}=\left\|\Lambda \widetilde{q}_{l}\right\|_{L^{2}}^{2}+A\left\|\widetilde{d}_{l}\right\|_{L^{2}}^{2}-\frac{2}{\bar{\nu}}\left(\widetilde{q}_{l}, \widetilde{d}_{l}\right) .
$$

For the sake of simplicity, we suppose here that $F=G=0, v=0$ and $K=0$. We now want a control $\left\|\Lambda q_{l}\right\|_{L^{2}}^{2}$ on e apply the operator $\Lambda$ to the first equation of (3.12), multiply by $\Lambda q_{l}$ and integrate over $\mathbb{R}^{N}$, so we obtain:

$$
\frac{1}{2} \frac{d}{d t}\left\|\Lambda q_{l}\right\|_{L^{2}}^{2}+\left(\Lambda^{2} d_{l}, \Lambda q_{l}\right)=0
$$

Moreover we have:

$$
\begin{aligned}
& \frac{1}{2} \frac{d}{d t}\left\|d_{l}\right\|_{L^{2}}^{2}+\bar{\nu}\left\|\Lambda d_{l}\right\|_{L^{2}}^{2}-\bar{\delta}\left(\Lambda q_{l}, d_{l}\right)+\bar{\kappa}\left(\Lambda\left(\phi * q_{l}\right), d_{l}\right)=0 . \\
& \frac{d}{d t}\left(\Lambda q_{l}, d_{l}\right)+\left\|\Lambda d_{l}\right\|_{L^{2}}^{2}-\bar{\delta}\left\|\Lambda q_{l}\right\|_{L^{2}}^{2}+\bar{\kappa}\left\|\phi * \Lambda q_{l}\right\|_{L^{2}}^{2}+\bar{\nu}\left(\Lambda^{2} d_{l}, \Lambda q_{l}\right)=0 .
\end{aligned}
$$

By linear combination of (3.22)-(3.23) we have:

$$
\frac{1}{2} \frac{d}{d t} f_{l}^{2}+\frac{1}{\bar{\nu}}\left\|\Lambda q_{l}\right\|_{L^{2}}^{2}+\left(A \bar{\nu}-\frac{1}{\bar{\nu}}\right)\left\|\Lambda d_{l}\right\|_{L^{2}}^{2}-A \bar{\delta}\left(\Lambda q_{l}, d_{l}\right)+A \bar{\kappa}\left(\Lambda\left(\phi * q_{l}\right), d_{l}\right)=0 .
$$

Moreover we have:

$$
\left|-A \bar{\delta}\left(\Lambda q_{l}, d_{l}\right)+A \bar{\kappa}\left(\Lambda\left(\phi * q_{l}\right), d_{l}\right)\right| \leq A\left(\bar{\delta}+\bar{\kappa}\|\widehat{\phi}\|_{L^{\infty}}\right)\left|\left(\Lambda q_{l}, d_{l}\right)\right|
$$

We have now in using Young inequalities for all $a>0$ :

$$
\left|\left(d_{l}, \Lambda q_{l}\right)\right| \leq \frac{a}{2}\left\|\Lambda q_{l}\right\|_{L^{2}}^{2}+\frac{1}{2 a}\left\|d_{l}\right\|_{L^{2}}^{2}
$$

So we get:

$$
\frac{1}{2} \frac{d}{d t} f_{l}^{2}+2^{2 l_{0}}\left(A \bar{\nu}-\frac{1}{\bar{\nu}}-\frac{1}{2 a}\right)\left\|d_{l}\right\|_{L^{2}}^{2}+\left(\frac{1}{\bar{\nu}}-\frac{a}{2}\right)\left\|\Lambda q_{l}\right\|_{L^{2}}^{2} . \leq 0
$$


So in choosing:

$$
a=\frac{1}{\bar{\nu} A} \quad \text { and } \quad A>\max \left(\frac{2}{\bar{\nu}}, 1\right)
$$

there exists a constant $\alpha$ such that for $l \geq l_{0}+1$ we have:

$$
\frac{1}{2} \frac{d}{d t} f_{l}^{2}+\alpha f_{l}^{2} \leq 0
$$

In the general case where $F, G, H, K$ and $v$ are not necessarily zero, we use a lemma of harmonic analysis in [15] to control the convection terms. We finally get:

$$
\begin{aligned}
\frac{1}{2} \frac{d}{d t} f_{l}^{2}+\left(\alpha+K V^{\prime}\right) f_{l}^{2} \lesssim \alpha_{l} f_{l} 2^{-l(s-1)}\left(\|(\widetilde{F}, \widetilde{G})\|_{\widetilde{B}^{s-1, s} \times B^{s-1}}\right. & \\
& \left.+V^{\prime}\|(\widetilde{q}, \widetilde{d})\|_{\widetilde{B}^{s-1, s} \times B^{s-1}}\right) .
\end{aligned}
$$

This finish the proof of (3.9) and (3.11).

\section{The damping effect}

We are now going to show that inequality (3.11) entails a decay for $q$ and $d$. In fact we get a parabolic decay for $d$, while $q$ has a behavior similar to a transport equation. Using $h_{l}^{2}=f_{l}^{2}+\delta^{2}$, integrating over $[0, t]$ and then having $\delta$ tend to 0 , we infer:

$$
\begin{aligned}
& f_{l}(t)+\alpha \min \left(2^{2 l}, 1\right) \int_{0}^{t} f_{l}(\tau) d \tau \\
& \leq f_{l}(0)+C 2^{-l(s-1)} \int_{0}^{t} \alpha_{l}(\tau)\|(\widetilde{F}(\tau), \widetilde{G}(\tau))\|_{\widetilde{B}^{s-1, s} \times B^{s}} d \tau \\
& \quad+\int_{0}^{t} V^{\prime}(\tau)\left(C 2^{-l(s-1)} \alpha_{l}(\tau)\|(\widetilde{q}, \widetilde{d})\|_{\tilde{B}^{s-1, s} \times B^{s}}-K f_{l}(\tau)\right) d \tau .
\end{aligned}
$$

Thanks to (3.10), we have in taking $K$ large enough :

$$
\sum_{l \in \mathbb{Z}}\left(C 2^{-l(s-1)} \alpha_{l}(\tau)\|(\widetilde{q}, \widetilde{d})\|_{\tilde{B}^{s-1, s} \times B^{s}}-K f_{l}(\tau)\right) \leq 0,
$$

In multiplying $\left(\underline{3.28)}\right.$ by $2^{l(s-1)}$ and in using the last inequality, we conclude after summation on $\mathbb{Z}$, that:

$$
\begin{array}{r}
\|\widetilde{q}(t)\|_{\tilde{B}^{s-1, s}}+\|\widetilde{d}\|_{\tilde{B}^{s-1}}+\alpha \int_{0}^{t}\|\widetilde{q}(\tau)\|_{\widetilde{B}^{s-1, s}} d \tau+\sum_{l \in \mathbb{Z}} \int_{0}^{t} \alpha 2^{l(s-1)} \min \left(2^{2 l}, 1\right)\left\|\widetilde{d}_{l}(\tau)\right\|_{L^{2}} d \tau \\
\quad \lesssim\left\|\left(\widetilde{q}_{0}, \widetilde{d}_{0}\right)\right\|_{\widetilde{B}^{s-1, s} \times B^{s-1}}+\int_{0}^{t}\|(\widetilde{F}, \widetilde{G})\|_{\widetilde{B}^{s-1, s} \times B^{s-1}} d \tau .
\end{array}
$$




\section{The smoothing effect}

Once stated the damping effect for $q$, it is easy to get the smoothing effect on $d$ by considering the last two equations where the term $\Lambda q$ is considered as a source term . Thanks to (3.29), it suffices to prove it for high frequencies only. We therefore suppose in this subsection that $l \geq l_{0}$ for a $l_{0}$ big enough.

We set $g_{l}=\left\|\widetilde{d}_{l}\right\|_{L^{2}}$ and in using the previous inequalities, we have:

$$
\frac{1}{2} \frac{d}{d t}\left\|\widetilde{d}_{l}\right\|_{L^{2}}^{2}+\bar{\nu}\left\|\Lambda \widetilde{d}_{l}\right\|_{L^{2}}^{2}-\bar{\delta}\left(\Lambda \widetilde{q}_{l}, \widetilde{d}_{l}\right)+\bar{\kappa}\left(\Lambda\left(\phi * \widetilde{q}_{l}\right), \widetilde{d}_{l}\right)=\widetilde{G}_{l} \cdot \widetilde{d}_{l}-K V^{\prime}(t)\left\|\widetilde{d}_{l}\right\|_{L^{2}}^{2} .
$$

We get finally with $\alpha>0$ :

$$
\frac{1}{2} \frac{d}{d t} g_{l}^{2}+\alpha 2^{2 l} g_{l}^{2} \leq g_{l}\left(\left\|\Lambda \widetilde{q}_{l}\right\|_{L^{2}}+\left\|\widetilde{G}_{l}\right\|_{L^{2}}\right)+g_{l} V^{\prime}(t)\left(C \alpha_{l} 2^{-l(s-1)}\|\widetilde{d}\|_{B^{s-1}}-K g_{l}\right) .
$$

We therefore get in using standard computations:

$$
\begin{array}{r}
\sum_{l \geq l_{0}} 2^{l(s-1)}\left\|\widetilde{d}_{l}(t)\right\|_{L^{2}}+\alpha \int_{0}^{t} \sum_{l \geq l_{0}} 2^{l(s+1)}\left\|\widetilde{d}_{l}(\tau)\right\|_{L^{2}} d \tau \leq\left\|d_{0}\right\|_{B^{s-1}}+\int_{0}^{t}\|\widetilde{G}(\tau)\|_{B^{s-1}} d \tau \\
\quad+\int_{0}^{t} \sum_{l \geq l_{0}} 2^{l s}\left\|\widetilde{q}_{l}(\tau)\right\|_{L^{2}}+C V(t) \sup _{\tau \in[0, t]}\left(\|\widetilde{d}(\tau)\|_{B^{s-1}}\right) .
\end{array}
$$

Using the above inequality and (3.29), we have:

$$
\begin{aligned}
\int_{0}^{t} \sum_{l \geq l_{0}} 2^{l(s+1)}\left\|\widetilde{d}_{l}(\tau)\right\|_{L^{2}} d \tau \lesssim(1+V(t))\left(\left\|q_{0}\right\|_{\widetilde{B}^{s-1, s}}+\left\|d_{0}\right\|_{B^{s-1}}\right) & \\
& +\int_{0}^{t}\left(\|\widetilde{F}(\tau)\|_{\widetilde{B}^{s-1, s}}+\|\widetilde{G}(\tau)\|_{B^{s-1}}\right) d \tau .
\end{aligned}
$$

Combining that last inequality (3.30) with (3.29), we achieve the proof of proposition 3.11

\subsection{Proof of theorem 1.1}

This section is devoted to the proof of the theorem 1.1. The principle of the proof is a very classical one. We want to construct a sequence $\left(q^{n}, u^{n}\right)_{n \in \mathbb{N}}$ of approximate solutions of the system $(S W)$, and we will use the proposition 3.11 to get some uniform bounds on $\left(q^{n}, u^{n}\right)_{n \in \mathbb{N}}$. We will conclude by stating some properties of compactness, which will guarantee that up to an extraction, $\left(q^{n}, u^{n}\right)_{n \in \mathbb{N}}$ converges to a solution $(q, u)$ of the system $(S W)$.

First step: Building the sequence $\left(q^{n}, u^{n}\right)_{n \in \mathbb{N}}$

We start with the construction of the sequence $\left(q^{n}, u^{n}\right)_{n \in \mathbb{N}}$, in this goal we use the Friedrichs operators $\left(J_{n}\right)_{n \in \mathbb{N}}$ defined by:

$$
J_{n} g=\mathcal{F}^{-1}\left(1_{B\left(\frac{1}{n}, n\right)} \widehat{g}\right),
$$


where $\mathcal{F}^{-1}$ is the inverse Fourier transform. Let us consider the approximate system:

$$
\left\{\begin{array}{l}
\partial_{t} q^{n}+J_{n}\left(J_{n} u^{n} \cdot \nabla J_{n} q^{n}\right)+\Lambda J_{n} d^{n}=F^{n} \\
\partial_{t} d^{n}+J_{n}\left(J_{n} u^{n} \cdot \nabla J_{n} d^{n}\right)-\bar{\nu} \Delta J_{n} d^{n}-\bar{\delta} \Lambda J_{n} q^{n}-\bar{\kappa} \phi * \Lambda J_{n} q^{n}=G^{n} \\
\partial_{t} \Omega^{n}-\bar{\nu} \Delta J_{n} \Omega^{n}=H^{n} \\
u^{n}=-\Lambda^{-1} \nabla d^{n}-\Lambda^{-1} \operatorname{div} \Omega^{n} \\
\left(q^{n}, d^{n}, \Omega^{n}\right)_{/ t=0}=\left(J_{n} q_{0}, J_{n} d_{0}, J_{n} \Omega_{0}\right)
\end{array}\right.
$$

with:

$$
\begin{aligned}
& F^{n}=-J_{n}\left(\left(J_{n} q^{n}\right) \operatorname{div} J_{n} u^{n}\right), \\
& G^{n}=J_{n} \Lambda^{-1} \operatorname{div}\left[\mathcal{A}\left(\varphi\left(\bar{\rho}\left(1+J_{n} q^{n}\right)\right), J_{n} u^{n}\right)+K\left(\varphi\left(\bar{\rho}\left(1+J_{n} q^{n}\right)\right) \nabla q^{n}\right],\right. \\
& H^{n}=J_{n} \Lambda^{-1} \operatorname{curl}\left[\mathcal{A}\left(\varphi\left(\bar{\rho}\left(1+J_{n} q^{n}\right)\right), J_{n} u^{n}\right)+K\left(\varphi\left(\bar{\rho}\left(1+J_{n} q^{n}\right)\right) \nabla q^{n}\right] .\right.
\end{aligned}
$$

where $\varphi$ is a smooth function verifying $\varphi(s)=s$ for $\frac{1}{n} \leq s \leq n$ and $\varphi \geq \frac{1}{4}$.

We want to show that (3.31) is only an ordinary differential equation in $L^{2} \times L^{2} \times L^{2}$. We can observe easily that all the source term in (3.31) turn out to be continuous in $L^{2} \times L^{2} \times L^{2}$. As a example, we consider the term $J_{n} \mathcal{A}\left(\varphi\left(\bar{\rho}\left(1+J_{n} q^{n}\right)\right), J_{n} u^{n}\right)$. We have then by Plancherel theorem:

$$
\begin{gathered}
\left\|J_{n}\left(\frac{\operatorname{div}\left(\mu\left(\varphi\left(\bar{\rho}\left(1+J_{n} q^{n}\right)\right) D J_{n} u^{n}\right)\right.}{\varphi\left(\bar{\rho}\left(1+J_{n} q^{n}\right)\right)}\right)\right\|_{L^{2}} \leq n \| \mu\left(\varphi\left(\bar{\rho}\left(1+J_{n} q^{n}\right)\right) D J_{n} u^{n} \|_{L^{2}}\right. \\
\times\left\|\frac{1}{\varphi\left(\bar{\rho}\left(1+J_{n} q^{n}\right)\right)}\right\|_{L^{\infty}} \\
\leq 4 M_{n} n^{2}\left\|u^{n}\right\|_{L^{2}}
\end{gathered}
$$

where $M_{n}=\| \mu\left(\varphi\left(\bar{\rho}\left(1+J_{n} q^{n}\right) \|_{L^{\infty}}\right.\right.$.

According to the Cauchy-Lipschitz theorem, a unique maximal solution exists in $C\left(\left[0, T_{n}\right) ; L^{2}\right)$ with $T_{n}>0$. Moreover, since $J_{n}=J_{n}^{2}$ we show that $\left(J_{n} q^{n}, J_{n} d^{n}, J_{n} \Omega^{n}\right)$ is also a solution and then by uniqueness we get that $\left(J_{n} q^{n}, J_{n} u^{n}\right)=\left(q^{n}, u^{n}\right)$. This implies that $\left(q^{n}, d^{n}, \Omega^{n}\right)$ is solution of the following system:

$$
\left\{\begin{array}{l}
\partial_{t} q^{n}+J_{n}\left(u^{n} \cdot \nabla q^{n}\right)+\Lambda d^{n}=F_{1}^{n} \\
\partial_{t} d^{n}+J_{n}\left(u^{n} \cdot \nabla d^{n}\right)-\bar{\nu} \Delta d^{n}-\bar{\delta} \Lambda q^{n}-\bar{\kappa} \phi * \Lambda q^{n}=G_{1}^{n} \\
\partial_{t} \Omega^{n}-\bar{\nu} \Delta \Omega^{n}=H_{1}^{n} \\
u^{n}=-\Lambda^{-1} \nabla d^{n}-\Lambda^{-1} \operatorname{div} \Omega^{n} \\
\left(q^{n}, d^{n}, \Omega^{n}\right)_{t=0}=\left(J_{n} q_{0}, J_{n} d_{0}, J_{n} \Omega_{0}\right)
\end{array}\right.
$$

and:

$$
\begin{aligned}
& F_{1}^{n}=-J_{n}\left(q^{n} \operatorname{div} u^{n}\right), \\
& G_{1}^{n}=J_{n} \Lambda^{-1} \operatorname{div}\left[\mathcal{A}\left(\varphi\left(\bar{\rho}\left(1+q^{n}\right)\right), u^{n}\right)+K\left(\varphi\left(\bar{\rho}\left(1+q^{n}\right)\right)\right],\right. \\
& H_{1}^{n}=J_{n} \Lambda^{-1} \operatorname{curl}\left[\mathcal{A}\left(\varphi\left(\bar{\rho}\left(1+q^{n}\right)\right), u^{n}\right)+K\left(\varphi\left(\bar{\rho}\left(1+q^{n}\right)\right)\right] .\right.
\end{aligned}
$$


And the system (3.32) is again an ordinary differential equation in $L_{n}^{2}$ with:

$$
L_{n}^{2}=\left\{g \in L^{2}\left(\mathbb{R}^{N}\right) / \operatorname{supp} \widehat{g} \subset B\left(\frac{1}{n}, n\right)\right\} .
$$

Due to the Cauchy-Lipschitz theorem again, a unique maximal solution exists in $C^{1}\left(\left[0, T_{n}^{\prime}\right) ; L_{n}^{2}\right)$ with $T_{n}^{\prime} \geq T_{n}>0$.

\section{Second step: Uniform estimates}

In this part, we want to get uniform estimates independent of $T$ on $\left\|\left(q^{n}, u^{n}\right)\right\|_{E_{T}^{\frac{N}{2}}}$ for all $T<T_{n}^{\prime}$. This will show that $T_{n}^{\prime}=+\infty$ by Cauchy-Lipchitz because the norms $\|\cdot\|_{E^{\frac{N}{2}}}$ and $L^{2}$ are equivalent on $L_{n}^{2}$. $E_{T}^{\frac{N}{2}}$ Let us set:

$$
\begin{aligned}
& E(0)=\left\|q_{0}\right\|_{\widetilde{B}^{\frac{N}{2}-1, \frac{N}{2}}}+\left\|u_{0}\right\|_{B^{\frac{N}{2}}}, \\
& E(q, u, t)=\|q\|_{L_{t}^{\infty}\left(\widetilde{B}^{\frac{N}{2}-1, \frac{N}{2}}\right)}+\|q\|_{L_{t}^{\infty}\left(B^{\frac{N}{2}-1}\right)}+\|q\|_{L_{t}^{1}\left(\widetilde{B}^{\frac{N}{2}+1, \frac{N}{2}}\right)}+\|q\|_{L_{t}^{\infty}\left(B^{\frac{N}{2}+1}\right)},
\end{aligned}
$$

and:

$$
\bar{T}_{n}=\sup \left\{t \in\left[0, T_{n}^{\prime}\right), E\left(q^{n}, u^{n}, t\right) \leq 3 C E(0)\right\}
$$

$C$ corresponds to the constant in the proposition 3.11 and as $C>1$ we have $3 C>1$ so by continuity we have $\bar{T}_{n}>0$.

We are going to prove that $\bar{T}_{n}=T_{n}^{\prime}$ for all $n \in \mathbb{N}$ and we will conclude that $\forall n \in \mathbb{N}$ $T_{n}^{\prime}=+\infty$. To achieve it, one can use the proposition 3.11 to the system (3.32) to obtain uniform bounds, so we get in setting $V_{n}(t)=\left\|u^{n}\right\|_{L_{T}^{1}\left(B^{\frac{N}{2}+1}\right)}$ :

$$
\begin{aligned}
\left\|\left(q^{n}, u^{n}\right)\right\|_{E_{T}^{\frac{N}{2}}} \leq C e^{C V_{n}(t)}\left(\left\|q_{0}\right\|_{\tilde{B}^{\frac{N}{2}-1, \frac{N}{2}}}\right. & +\left\|u_{0}\right\|_{B^{\frac{N}{2}}}+\int_{0}^{T} e^{-C V_{n}(\tau)}\left(\left\|F_{1}^{n}(\tau)\right\|_{\widetilde{B}^{\frac{N}{2}-1, \frac{N}{2}}}\right. \\
& \left.\left.+\left\|G_{1}^{n}(\tau)\right\|_{B^{\frac{N}{2}-1}}+\left\|H_{1}^{n}(\tau)\right\|_{B^{\frac{N}{2}-1}}\right) d \tau .\right)
\end{aligned}
$$

Therefore, it is only a matter of proving appropriate estimates for $F_{1}^{n}, G_{1}^{n}$ and $H_{1}^{n}$ in using properties of continuity on the paraproduct.

We estimate now $\left\|F_{1}^{n}\right\|_{L_{T}^{1}\left(\widetilde{B}^{\left.\frac{N}{2}-1, \frac{N}{2}\right)}\right.}$ in using proposition 2.6 and 2.7;

$$
\left\|F_{1}^{n}\right\|_{L_{T}^{1}\left(B^{\left.\frac{N}{2}-1, \frac{N}{2}\right)}\right.} \leq C\left\|q^{n}\right\|_{L_{T}^{\infty}\left(B^{\left.\frac{N}{2}-1, \frac{N}{2}\right)}\right.}\left\|\operatorname{div} u^{n}\right\|_{L_{T}^{1}\left(B^{\frac{N}{2}}\right)},
$$

We now want to estimate $G_{1}^{n}$ :

$$
\left\|\mathcal{A}\left(\varphi\left(\bar{\rho}\left(1+q^{n}\right)\right), u^{n}\right)\right\|_{L_{T}^{1}\left(B^{\frac{N}{2}-1}\right)} \leq C\left\|u^{n}\right\|_{L_{T}^{1}\left(B^{\frac{N}{2}+1}\right)}\left\|q^{n}\right\|_{L_{T}^{\infty}\left(B^{\frac{N}{2}}\right)}\left(1+\left\|q^{n}\right\|_{L_{T}^{\infty}\left(B^{\frac{N}{2}}\right)}\right),
$$

We can verify that $K$ fulfills the hypothesis of the proposition 2.7, so we get:

$$
\| K\left(\varphi\left(\bar{\rho}\left(1+q^{n}\right)\right) \nabla q^{n}\left\|_{L_{T}^{1}\left(B^{\frac{N}{2}-1}\right)} \leq C\right\| q^{n}\left\|_{L_{T}^{2}\left(B^{\frac{N}{2}}\right)}^{2}\right\| q^{n} \|_{L_{T}^{\infty}\left(\widetilde{B}^{\frac{N}{2}-1, \frac{N}{2}}\right)},\right.
$$


Moreover we recall that according to proposition 2.6.

$$
\left\|q^{n}\right\|_{L_{T}^{2}\left(B^{\frac{N}{2}}\right)}^{2} \leq\left\|q^{n}\right\|_{L_{T}^{\infty}\left(\widetilde{B}^{\frac{N}{2}-1, \frac{N}{2}}\right)}\left\|q^{n}\right\|_{L_{T}^{1}\left(\widetilde{B}^{\frac{N}{2}+1, \frac{N}{2}}\right)} .
$$

We proceed similarly to estimate $\left\|H_{1}^{n}\right\|_{L_{T}^{1}\left(B^{\frac{N}{2}-1}\right)}$ and finally we have:

$$
\begin{aligned}
\left\|F_{1}^{n}\right\|_{L_{T}^{1}\left(B^{\frac{N}{2}-1}\right)}+\left\|G_{1}^{n}\right\|_{L_{T}^{1}\left(B^{\frac{N}{2}-1}\right)}+\left\|H_{1}^{n}\right\|_{L^{1}\left(B_{T}^{\frac{N}{2}-1}\right)} \leq 2 C\left(E^{2}\left(q^{n}, u^{n}, T\right)\right. \\
\left.+E^{3}\left(q^{n}, u^{n}, T\right)\right),
\end{aligned}
$$

whence:

$$
\left\|\left(q^{n}, u^{n}\right)\right\|_{E_{T}^{\frac{N}{2}}} \leq C e^{C^{2} 3 E(0)} E(0)(1+18 C E(0)(1+3 E(0))),
$$

We want now to get:

$$
e^{3 C^{2} E(0)}(1+18 C E(0)(1+3 E(0))) \leq 2
$$

for this it suffices choose $E(0)$ small enough, let $E(0)<\varepsilon$ such that:

$$
1+18 C E(0)(1+3 E(0)) \leq \frac{3}{2} \text { and } e^{3 C^{2} E(0)} \leq \frac{4}{3} .
$$

So we get $\bar{T}_{n}=T_{n}^{\prime}$, indeed we have shown that $\forall T$ such that $T<\bar{T}_{n}$ :

$$
E\left(q^{n}, u^{n}, T\right) \leq 2 C E(0) .
$$

Then we have $\bar{T}_{n}=T_{n}^{\prime}$, because if $\bar{T}_{n}<T_{n}^{\prime}$ we have seen that $E\left(q^{n}, u^{n}, \bar{T}_{n}\right) \leq 2 C E(0)$ and so by continuity for $\bar{T}_{n}+\varepsilon$ with $\varepsilon$ small enough we obtain again $E\left(q^{n}, u^{n}, \bar{T}_{n}+\varepsilon\right) \leq 3 C E(0)$ and stands in contradiction with the definition of $\bar{T}_{n}$.

So if $\bar{T}_{n}=T_{n}^{\prime}<+\infty$ we have seen that:

$$
E\left(q^{n}, u^{n}, T_{n}^{\prime}\right) \leq 3 C E(0) .
$$

As $\left\|q_{n}\right\|_{L_{T_{n}^{\prime}}^{\infty}\left(\widetilde{B}^{\frac{N}{2}}\right)}<+\infty$ and $\left\|u_{n}\right\|_{L_{T_{n}^{\prime}}^{\infty}\left(\widetilde{B}^{\frac{N}{2}-1}\right)}<+\infty$, it implies that $\left\|q_{n}\right\|_{L_{T_{n}^{\prime}}^{\infty}\left(L_{n}^{2}\right)}<+\infty$ and $\left\|u_{n}\right\|_{L_{T_{n}^{\prime}}^{\infty}\left(L_{n}^{2}\right)}^{T_{n}^{\prime}}<+\infty$, so by Cauchy-Lipschitz theorem, one may continue the solution beyond $T_{n}^{\prime}$ which contradicts the definition of $T_{n}^{\prime}$.

Finally the approximate solution $\left(q^{n}, u^{n}\right)_{n \in \mathbb{N}}$ is global in time.

\section{Second step: existence of a solution}

In this part, we shall show that, up to an extraction, the sequence $\left(q^{n}, u^{n}\right)_{n \in \mathbb{N}}$ converges in $\mathcal{D}^{\prime}\left(\mathbb{R}^{+} \times \mathbb{R}^{N}\right)$ to a solution $(q, u)$ of $(S W)$ which has the desired regularity properties. The proof lies on compactness arguments. To start with, we show that the time first derivative of $\left(q^{n}, u^{n}\right)$ is uniformly bounded in appropriate spaces. This enables us to apply Ascoli's theorem and get the existence of a limit $(q, u)$ for a subsequence. Now, the uniform bounds of the previous part provide us with additional regularity and convergence properties so that we may pass to the limit in the system.

It is convenient to split $\left(q^{n}, u^{n}\right)$ into the solution of a linear system with initial data 
$\left(q_{n}, u_{n}\right)$ and forcing term, and the discrepancy to that solution.

More precisely, we denote by $\left(q_{L}^{n}, u_{L}^{n}\right)$ the solution to:

$$
\begin{aligned}
& \partial_{t} q_{L}^{n}+\operatorname{div} u_{L}^{n}=0 \\
& \partial_{t} u_{L}^{n}-\mathcal{A} u_{L}^{n}+\nabla q_{L}^{n}=0 \\
& \left(q_{L}^{n}, v_{L}^{n}\right)_{/ t=0}=\left(J_{n} q_{0}, J_{n} u_{0}\right)
\end{aligned}
$$

where: $\mathcal{A}=\bar{\mu} \Delta+(\bar{\lambda}+\bar{\mu}) \nabla \operatorname{div}$ and we set $\left(\bar{q}^{n}, \bar{u}^{n}\right)=\left(q^{n}-q_{L}^{n}, u^{n}-u_{L}^{n}\right)$.

Obviously, the definition of $\left(q_{L}^{n}, v_{L}^{n}\right)_{/ t=0}$ entails:

$$
\left(q_{L}^{n}\right)_{/ t=0} \rightarrow q_{0} \text { in } \widetilde{B}^{\frac{N}{2}-1, \frac{N}{2}},\left(u_{L}^{n}\right)_{/ t=0} \rightarrow u_{0} \text { in } \tilde{B}^{\frac{N}{2}-1} .
$$

The proposition 2.9 insures that $\left(q_{L}^{n}, u_{L}^{n}\right)$ converges to the solution $\left(q_{L}, u_{L}\right)$ of the linear system associated to (3.33) in $E^{\frac{N}{2}}$. We now have to prove the convergence of $\left(\bar{q}^{n}, \bar{u}^{n}\right)$. This is of course a trifle more difficult and requires compactness results. Let us first state the following lemma.

Lemma $\left.1\left(q^{n}, u^{n}\right)\right)_{n \in \mathbb{N}}$ is uniformly bounded in $C^{\frac{1}{2}}\left(\mathbb{R}^{+} ; B^{\frac{N}{2}-1}\right) \times\left(C^{\frac{1}{4}}\left(\mathbb{R}^{+} ; B^{\frac{N}{2}-\frac{3}{2}}\right)\right)^{N}$.

\section{Proof:}

In all the proof, we will note u.b for uniformly bounded.

We first prove that $\frac{\partial}{\partial t} q^{n}$ is u.b in $L^{2}\left(\mathbb{R}^{+}, B^{\frac{N}{2}-1}\right)$, which yields the desired result for $q^{n}$. Let us observe that $q^{n}$ verifies the following equation

$$
\frac{\partial}{\partial t} q^{n}=\operatorname{div} u^{n}-J_{n}\left(u^{n} \cdot \nabla q^{n}\right)-J_{n}\left(q^{n} \operatorname{div} u^{n}\right) .
$$

According to the first part, $\left(u_{n}\right)_{n \in \mathbb{N}}$ is u.b in $L^{2}\left(B^{\frac{N}{2}}\right)$, so we can conclude that $\frac{\partial}{\partial t} q^{n}$ is u.b in $L^{2}\left(B^{\frac{N}{2}-1}\right)$. Indeed we have:

$$
\begin{aligned}
& \left\|J_{n}\left(q^{n} \operatorname{div} u^{n}\right)\right\|_{L^{2}\left(B^{\frac{N}{2}-1}\right)} \leq\left\|u^{n}\right\|_{L^{2}\left(B^{\frac{N}{2}}\right)}\left\|q^{n}\right\|_{L^{\infty}\left(B^{\frac{N}{2}}\right)} \\
& \left\|J_{n}\left(u^{n} \cdot \nabla q^{n}\right)\right\|_{L^{2}\left(B^{\frac{N}{2}-1}\right)} \leq\left\|u^{n}\right\|_{L^{2}\left(B^{\frac{N}{2}}\right)}\left\|q^{n}\right\|_{L^{\infty}\left(B^{\frac{N}{2}}\right)} .
\end{aligned}
$$

And we recall that we use the fact that $\widetilde{B}^{\frac{N}{2}-1, \frac{N}{2}} \hookrightarrow B^{\frac{N}{2}}$.

Let us prove now that $\frac{\partial}{\partial t} d^{n}$ is u.b in $L^{\frac{4}{3}}\left(B^{\frac{N}{2}-\frac{3}{2}}\right)+L^{4}\left(B^{\frac{N}{2}-\frac{3}{2}}\right)$ and that $\partial_{t} \Omega^{n}$ is u.b in $L^{\frac{4}{3}}\left(B^{\frac{N}{2}-\frac{3}{2}}\right)$ (which gives the required result for $u^{n}$ in using the relation $u^{n}=-\Lambda^{-1} \nabla d^{n}-$ $\left.\Lambda^{-1} \operatorname{div} \Omega^{n}\right)$.

Let us recall that:

$$
\begin{aligned}
& \frac{\partial}{\partial t} d^{n}=J_{n}\left(u^{n} \cdot \nabla d^{n}\right)+J_{n} \Lambda^{-1} \operatorname{div}\left[\mathcal{A}\left(\varphi\left(\bar{\rho}\left(1+q^{n}\right)\right), u^{n}\right)\right.\left.+J_{n}\left(K\left(\varphi\left(\bar{\rho}\left(1+q^{n}\right)\right)\right) \nabla q^{n}\right)\right] \\
&+\bar{\nu} \Delta d^{n}+\bar{\delta} \Lambda q^{n}-\bar{\kappa} \phi * \Lambda q^{n}, \\
& \frac{\partial}{\partial t} \Omega^{n}=J_{n} \Lambda^{-1} \operatorname{curl}\left[\mathcal{A}\left(\varphi\left(\bar{\rho}\left(1+q^{n}\right)\right), u^{n}\right)+J_{n}\left(K\left(\varphi\left(\bar{\rho}\left(1+q^{n}\right)\right) \nabla q^{n}\right)\right)\right]+\bar{\mu} \Delta \Omega^{n} .
\end{aligned}
$$


Results of step one and an interpolation argument yield uniform bounds for $u^{n}$ in $L^{\infty}\left(B^{\frac{N}{2}-1}\right) \cap L^{\frac{4}{3}}\left(B^{\frac{N}{2}+\frac{1}{2}}\right)$, we infer in proceeding as for $\frac{\partial}{\partial t} q^{n}$ that:

$$
A_{n}=J_{n}\left(u^{n} \cdot \nabla d^{n}\right)+J_{n} \Lambda^{-1} \operatorname{div}\left[\mathcal{A}\left(\varphi\left(\bar{\rho}\left(1+q^{n}\right)\right), u^{n}\right)+J_{n}\left(K\left(\varphi\left(\bar{\rho}\left(1+q^{n}\right)\right)\right) \nabla q^{n}\right)\right]+\bar{\nu} \Delta d^{n}
$$

is u.b in $L^{\frac{4}{3}}\left(B^{\frac{N}{2}-\frac{3}{2}}\right)$.

Using the bounds for $q^{n}$ in $L^{2}\left(B^{\frac{N}{2}}\right) \cap L^{\infty}\left(\widetilde{B}^{\frac{N}{2}-1, \frac{N}{2}}\right)$, we get $q^{n}$ u.b in $L^{4}\left(B^{\frac{N}{2}-\frac{1}{2}}\right)$ in using proposition 2.6. We thus have $J_{n}\left(K\left(\varphi\left(\bar{\rho}\left(1+q^{n}\right)\right) \nabla q^{n}\right.\right.$ u.b in $L^{\frac{4}{3}}\left(B^{\frac{N}{2}-\frac{3}{2}}\right)$.

Using the bounds for $u^{n}$ in $L^{\infty}\left(B^{\frac{N}{2}-1}\right) \cap L^{\frac{4}{3}}\left(B^{\frac{N}{2}+\frac{1}{2}}\right)$ we finally get $A_{n}$ is u.b in $L^{\frac{4}{3}}\left(B^{\frac{N}{2}-\frac{3}{2}}\right)$. To conclude $\phi * \Lambda q^{n}$ is u.b in $L^{4}\left(B^{\frac{N}{2}-\frac{3}{2}}\right)$, so $\frac{\partial}{\partial t} d^{n}$ is u.b in $L^{\frac{4}{3}}\left(B^{\frac{N}{2}-\frac{3}{2}}\right)+L^{4}\left(B^{\frac{N}{2}-\frac{3}{2}}\right)$.

The case of $\frac{\partial}{\partial t} \Omega^{n}$ goes along the same lines. As the terms corresponding to $\Lambda q^{n}$ and $\phi * \Lambda \bar{q}^{n}$ do not appear, we simply get $\partial_{t} \Omega^{n}$ u.b in $L^{\frac{4}{3}}\left(B^{\frac{N}{2}-\frac{3}{2}}\right)$.

We can now turn to the proof of the existence of a solution and using Ascoli theorem to get strong convergence. We proceed similarly to the theorem of Aubin-Lions.

Theorem 3.4 Let $X$ a compact metric space and $Y$ a complete metric space. Let $A$ be an equicontinuous part of $C(X, Y)$. Then we have the two equivalent proposition:

1. A is relatively compact in $C(X, Y)$

2. $A(x)=\{f(x) ; f \in A\}$ is relatively compact in $Y$

We need to localize because we have some result of compactness for the local Sobolev space. Let $\left(\chi_{p}\right)_{p \in \mathbb{N}}$ be a sequence of $C_{0}^{\infty}\left(\mathbb{R}^{N}\right)$ cut-off functions supported in the ball $B(0, p+1)$ of $\mathbb{R}^{N}$ and equal to 1 in a neighborhood of $B(0, p)$.

For any $p \in \mathbb{N}$, lemma 1 tells us that $\left(\left(\chi_{p} q^{n}, \chi_{p} u^{n}\right)\right)_{n \in \mathbb{N}}$ is uniformly equicontinuous in $C\left(\mathbb{R}^{+} ; B^{\frac{N}{2}-1} \times\left(B^{\frac{N}{2}-\frac{3}{2}}\right)^{N}\right)$. In using Ascoli's theorem we just need to show that $\left(\left(\chi_{p} q^{n}(t, \cdot), \chi_{p} u^{n}\right)(t, \cdot)\right)_{n \in \mathbb{N}}$ is relatively compact in $B^{\frac{N}{2}-1} \times\left(B^{\frac{N}{2}-\frac{3}{2}}\right)^{N} \forall t \in[0, p]$.

Let us observe now that the application $u \rightarrow \chi_{p} u$ is compact from $\widetilde{B}^{\frac{N}{2}-1, \frac{N}{2}}=B^{\frac{N}{2}} \cap B^{\frac{N}{2}-1}$ into $\dot{H}^{\frac{N}{2}-1}$, and from $B^{\frac{N}{2}-1} \cap B^{\frac{N}{2}-\frac{3}{2}}$ into $\dot{H}^{\frac{N}{2}-\frac{3}{2}}$.

After we apply Ascoli's theorem to the family $\left(\left(\chi_{p} q^{n}, \chi_{p} u^{n}\right)\right)_{n \in \mathbb{N}}$ on the time interval $[0, p]$. We then use Cantor's diagonal process. This finally provides us with a distribution $(q, u)$ belonging to $C\left(\mathbb{R}^{+} ; \dot{H}^{\frac{N}{2}-1} \times\left(\dot{H}^{\frac{N}{2}-\frac{3}{2}}\right)^{N}\right)$ and a subsequence (which we still denote by $\left(q^{n}, u^{n}\right)_{n \in \mathbb{N}}$ such that, for all $p \in \mathbb{N}$, we have:

$$
\left(\chi_{p} q^{n}, \chi_{p} u^{n}\right) \rightarrow_{n \mapsto+\infty}\left(\chi_{p} q, \chi_{p} u\right) \text { in } C\left([0, p] ; \dot{H}^{\frac{N}{2}-1} \times\left(\dot{H}^{\frac{N}{2}-\frac{3}{2}}\right)^{N}\right)
$$

This obviously entails that $\left(q^{n}, u^{n}\right)$ tends to $(q, u)$ in $\mathcal{D}^{\prime}\left(\mathbb{R}^{+} \times \mathbb{R}^{N}\right)$.

Coming back to the uniform estimates of step one, we moreover get that $(q, u)$ belongs to:

$$
L^{1}\left(\widetilde{B}^{\frac{N}{2}-1, \frac{N}{2}} \times\left(B^{\frac{N}{2}+1}\right)^{N}\right) \cap L^{\infty}\left(\widetilde{B}^{\frac{N}{2}-1, \frac{N}{2}} \times\left(B^{\frac{N}{2}+1}\right)^{N}\right)
$$

and to $C^{\frac{1}{2}}\left(\mathbb{R}^{+} ; B^{\frac{N}{2}-1}\right) \times\left(C^{\frac{1}{4}}\left(\mathbb{R}^{+} ; B^{\frac{N}{2}-\frac{3}{2}}\right)^{N}\right)$. Obviously, we have the bounds provided of the firts step. 
Let us now prove that $(q, u)$ solves the system $(S W)$, we first recall that $\left(q^{n}, u^{n}\right)$ solves the following system:

$$
\left\{\begin{aligned}
\partial_{t} q^{n}+J_{n}\left(u^{n} \cdot \nabla q^{n}\right)+\operatorname{div} u^{n}=-J_{n}\left(q^{n} \operatorname{div} u^{n}\right) & \\
\partial_{t} u^{n}-\bar{\nu} \Delta u^{n}+\bar{\delta} \nabla q^{n}-\bar{\kappa} \phi * \nabla q^{n}+J_{n}\left(u^{n} \cdot \nabla u^{n}\right)+J_{n}( & K\left(\varphi\left(\bar{\rho}\left(1+q^{n}\right)\right) \nabla q^{n}\right) \\
& +J_{n}\left(\mathcal{A}\left(\varphi\left(\bar{\rho}\left(1+q^{n}\right)\right), u^{n}\right)\right)=0
\end{aligned}\right.
$$

The only problem is to pass to the limit in $\mathcal{D}^{\prime}\left(\mathbb{R}^{+} \times \mathbb{R}^{N}\right)$ in the non linear terms. This can be done by using the convergence results coming from the uniform estimates (3.34). As it is just a matter of doing tedious verifications, we show as a example the case of the term $J_{n}\left(K\left(\varphi\left(\bar{\rho}\left(1+q^{n}\right)\right)\right) \nabla q^{n}\right)$ and $J_{n}\left(\mathcal{A}\left(\varphi\left(\bar{\rho}\left(1+q^{n}\right)\right), u^{n}\right)\right)$.

We decompose:

$$
J_{n}\left(K\left(\varphi\left(\bar{\rho}\left(1+q^{n}\right)\right)\right) \nabla q^{n}\right)-K\left(\rho^{n}\right) \nabla q^{n}=J_{n}\left(K\left(\varphi\left(\bar{\rho}\left(1+q^{n}\right)\right)\right) \nabla q^{n}\right)-K(\varphi(\bar{\rho}(1+q))) \nabla q .
$$

(Note that for $n$ big enough, we have $K\left(\varphi\left(\bar{\rho}\left(1+q^{n}\right)\right)\right)=K\left(\rho^{n}\right)$ as we control $\left\|\rho^{n}\right\|_{L^{\infty}}$ and $\left.\left\|\frac{1}{\rho^{n}}\right\|_{L^{\infty}}\right)$. Next we have:

$$
\begin{array}{r}
J_{n}\left(K\left(\varphi\left(\bar{\rho}\left(1+q^{n}\right)\right)\right) \nabla q^{n}\right)-K(\varphi(\bar{\rho}(1+q))) \nabla q=J_{n} A_{n}+\left(J_{n}-I\right) K(\varphi(\bar{\rho}(1+q))) \nabla q, \\
\text { where } A_{n}=K\left(\varphi\left(\bar{\rho}\left(1+q^{n}\right)\right)\right) \nabla q^{n}-K(\varphi(\bar{\rho}(1+q))) \nabla q .
\end{array}
$$

We have then $\left(J_{n}-I\right) K(\varphi(\bar{\rho}(1+q))) \nabla q$ tends to zero as $n \rightarrow+\infty$ due to the property of $J_{n}$ and the fact that $K(\varphi(\bar{\rho}(1+q))) \nabla q$ belongs to $L^{\infty}\left(B^{\frac{N}{2}-1}\right) \hookrightarrow L^{\infty}\left(L^{q}\right)$ for some $q \geq 2$. Choose $\psi \in C_{0}^{\infty}\left([0, T) \times \mathbb{R}^{N}\right)$ and $\varphi^{\prime} \in C_{0}^{\infty}\left([0, T) \times \mathbb{R}^{N}\right)$ such that $\varphi^{\prime}=1$ on $\operatorname{supp} \psi$, we have:

$$
\left|<\left(J_{n}-I\right) K(\varphi(\bar{\rho}(1+q))) \nabla q, \psi>\right| \leq\left\|\varphi^{\prime} K(\varphi(\bar{\rho}(1+q))) \nabla q\right\|_{L^{\infty}\left(L^{2}\right)}\left\|\left(J_{n}-I\right) \psi\right\|_{L^{2}},
$$

because $L_{l o c}^{q} \hookrightarrow L_{l o c}^{2}$ and we conclude by the fact that $\left\|\left(J_{n}-I\right) \psi\right\|_{L^{2}} \rightarrow 0$ as $n$ tends to $+\infty$.

Next:

$$
<J_{n} A_{n}, \psi>=I_{n}^{1}+I_{n}^{2}
$$

with:

$$
\begin{aligned}
& I_{n}^{1}=<\left(K\left(\varphi\left(\bar{\rho}\left(1+q^{n}\right)\right)\right)-K(\varphi(\bar{\rho}(1+q)))\right) \nabla q^{n}, J_{n} \psi> \\
& I_{n}^{2}=<K(\varphi(\bar{\rho}(1+q))) \nabla\left(q^{n}-q\right), J_{n} \psi>.
\end{aligned}
$$

We have then:

$$
I_{n}^{1} \leq\left\|\varphi^{\prime} q^{n}\right\|_{L^{\infty}\left(B^{\frac{N}{2}}\right)}\left\|\varphi^{\prime}\left(q^{n}-q\right)\right\|_{L^{\infty}\left(\dot{H}^{\frac{N}{2}-1}\right)}\|\psi\|_{L^{\infty}},
$$

Indeed we just use the fact that $\varphi^{\prime} B^{\frac{N}{2}-1}$ and $\varphi^{\prime} \dot{H}^{\frac{N}{2}-1}$ are embedded in $L^{2}$. Next we conclude as we have seen that $q^{n} \rightarrow_{n \rightarrow+\infty} q$ in $C_{l o c}\left(H_{l o c}^{\frac{N}{2}-1}\right)$. So we obtain:

$$
I_{n}^{1} \rightarrow_{n \rightarrow+\infty} 0 \text { in } \mathcal{D}^{\prime}\left(\left(0, T^{*}\right) \times \mathbb{R}^{N}\right) .
$$

We proceed similarly for $I_{n}^{2}$, indeed we have:

$$
I_{n}^{2}=<\varphi^{\prime}\left(q^{n}-q\right), \varphi^{\prime} \operatorname{div}\left(K(\varphi(\bar{\rho}(1+q))) J_{n} \psi\right)>
$$


and we have $K(\varphi(\bar{\rho}(1+q))) J_{n} \psi \in L^{\infty}\left(B^{\frac{N}{2}}\right)$ so:

$$
I_{n}^{2} \leq\left\|\varphi^{\prime}\left(q^{n}-q\right)\right\|_{L^{\infty}\left(\dot{H}^{\frac{N}{2}-1}\right)}\left\|K(\varphi(\bar{\rho}(1+q))) J_{n} \psi\right\|_{L^{\infty}\left(B^{\frac{N}{2}}\right)} .
$$

We conclude then that:

$$
I_{n}^{2} \rightarrow_{n \rightarrow+\infty} 0 \text { in } \mathcal{D}^{\prime}\left(\left(0, T^{*}\right) \times \mathbb{R}^{N}\right) .
$$

We concentrate us now on the term $J_{n}\left(\mathcal{A}\left(\varphi\left(\bar{\rho}\left(1+q^{n}\right)\right), u^{n}\right)\right)$. Let $\varphi^{\prime} \in C_{0}^{\infty}\left(\mathbb{R}^{+} \times \mathbb{R}^{N}\right)$ and $p \in \mathbb{N}$ be such that $\operatorname{supp} \varphi^{\prime} \subset[0, p] \times B(0, p)$. We use the decomposition for $n$ big enough:

$$
\begin{aligned}
\varphi^{\prime} J_{n} \mathcal{A}\left(\varphi\left(\bar{\rho}\left(1+q^{n}\right)\right), u^{n}\right)-\varphi^{\prime} \mathcal{A}(\rho, u)=\varphi^{\prime} \chi_{p} \mathcal{A}\left(\varphi\left(\bar{\rho}\left(1+q^{n}\right)\right), \chi_{p}\left(u^{n}-u\right)\right) \\
\left.+\varphi^{\prime} \mathcal{A}\left(\chi_{p} \varphi\left(\bar{\rho}\left(1+q^{n}\right)\right)-\chi_{p} \bar{\rho}(1+q)\right), u\right) .
\end{aligned}
$$

According to the uniform estimates and (3.34), $\chi_{p}\left(u^{n}-u\right)$ tends to 0 in $L^{1}\left([0, p] ; \dot{H}^{\frac{N}{2}+1}\right)$ by interpolation so that the first term tends to 0 in $L^{1}\left(\dot{H}^{\frac{N}{2}-1}\right)$ and we conclude for the second term in remarking that $\frac{\varphi}{\rho_{n}}$ tends to $\frac{\varphi}{\rho}$ as $\rho_{n}$ in $L^{\infty}\left(L^{\infty} \cap \dot{H}^{\frac{N}{2}}\right)$.

The other nonlinear terms can be treated in the same way.

\subsection{Proof of the uniqueness in the critical case}

Theorem 3.5 Let $N \geq 2$, and $\left(q_{1}, u_{1}\right)$ and $\left(q_{2}, u_{2}\right)$ be solutions of $(S W)$ with the same data $\left(q_{0}, u_{0}\right)$ on the time interval $\left[0, T^{*}\right)$. Assume that for $i=1,2$ :

$$
\left(q_{i}, u_{i}\right) \in C\left(\left[0, T^{*}\right), B_{N, 1}^{1}\right) \quad \text { and } \quad u_{i} \in\left(C\left(\left[0, T^{*}\right), B_{N, 1}^{0}\right) \cap L_{l o c}^{1}\left(\left[0, T^{*}\right), B_{N, 1}^{2}\right)\right)^{N} .
$$

There exists a constant $\alpha>0$ depending only on $N$ and physical constants such that if:

$$
\left\|q_{1}\right\|_{\widetilde{L}_{T^{*}}^{\infty}\left(B_{N, 1}^{1}\right)} \leq \alpha
$$

then $\left(q_{1}, u_{1}\right)=\left(q_{2}, u_{2}\right)$ on $\left[0, T^{*}\right)$.

Let $\left(q_{1}, u_{1}\right),\left(q_{2}, u_{2}\right)$ belong to $E^{\frac{N}{2}}$ with the same initial data, we set $(\delta q, \delta u)=\left(q_{2}-\right.$ $\left.q_{1}, u_{2}-u_{1}\right)$. We can then write the system $(S W)$ as follows:

$$
\left\{\begin{array}{l}
\frac{\partial}{\partial t} \delta q+u_{2} \cdot \nabla \delta q=H_{1} \\
\frac{\partial}{\partial t} \delta u-\bar{\nu} \Delta \delta u=H_{2}
\end{array}\right.
$$

with:

$$
\begin{aligned}
& H_{1}=-\operatorname{div} \delta u-\delta u \cdot \nabla q_{1}-\delta q \operatorname{div} u_{2}-q_{1} \operatorname{div} u \\
& H_{2}=-\bar{\delta} \nabla \delta q-\bar{\kappa} \phi * \nabla \delta q-u_{2} \cdot \nabla \delta u-\delta u \cdot \nabla u_{1}+\mathcal{A}\left(q_{1}, \delta u\right)+\mathcal{A}\left(\delta q, u_{2}\right) .
\end{aligned}
$$

Due to the term $\delta u \cdot \nabla q^{1}$ in the right-hand side of the first equation, we loose one derivative when estimating $\delta q$ : one only gets bounds in $L^{\infty}\left(B_{N, 1}^{0}\right)$. 
Now, the right hand-side of the second equation contains a term of type $\mathcal{A}\left(\delta q, u_{2}\right)$ so that the loss of one derivative for $\delta q$ entails a loss of one derivative for $\delta u$. Therefore, getting bounds in:

$$
C\left(\mathbb{R}^{+} ; B_{N, 1}^{-1}\right) \cap L^{1}\left(\mathbb{R}^{+} ; B_{N, 1}^{1}\right)
$$

for $\delta u$ is the best that one can hope. If enough regularity were available, we would not have to worry about this loss of derivative. But in the present case, the above heuristic fails because we have reached some limit cases for the product laws. Indeed, a term such as $\delta u \cdot \nabla u_{1}$ cannot be estimated properly: the product does not map $B_{N, 1}^{0} \times B_{N, 1}^{0}$ into $B_{N, 1}^{-1}$ but in the somewhat larger space $B_{N, \infty}^{-1}$. At this point, we could try instead to get bounds for $\delta u$ in:

$$
C\left(\left[0, T^{*}\right) ; B_{N, \infty}^{-1}\right) \cap L_{l o c}^{1}\left(\left[0, T^{*}\right) ; B_{N, \infty}^{1}\right),
$$

but we then have to face the lack of control on $\delta u$ in $L^{1}\left(0, T ; L^{\infty}\right)$ (because in contrast with $B_{N, 1}^{1}$, the space $B_{N, \infty}^{1}$ is not imbedded in $\left.L^{\infty}\right)$ so that we run into troubles when estimating $\delta u \cdot \nabla q_{1}$. The key to that difficulty relies on the following logarithmic interpolation inequality (see the proposition 2.5):

$$
\|u\|_{L_{T}^{1}\left(B_{N, 1}^{1}\right)} \lesssim\|u\|_{\widetilde{L}_{T}^{1}\left(B_{N, \infty}^{1}\right)} \log \left(e+\frac{\|u\|_{\widetilde{L}_{T}^{1}\left(B_{N, \infty}^{0}\right)}+\|u\|_{\widetilde{L}_{T}^{1}\left(B_{N, \infty}^{2}\right)}}{\left.\|u\|_{\widetilde{L}_{T}^{1}\left(B_{N, \infty}^{1}\right)}\right)},\right.
$$

and a well-known generalization of Grönwall the Osgood's lemma (see [14]) that we recall.

Lemma 2 Let $F$ be a measurable positive function and $\gamma$ a positive locally integrable function, each defined on the domain $\left[t_{0}, t_{1}\right]$. Let $\mu:[0,+\infty) \rightarrow[0,+\infty)$ be a continuous nondecreasing function, with $\mu(0)=0$. Let $a \geq 0$, and assume that for all $t \in\left[t_{0}, t_{1}\right]$,

$$
F(t) \leq a+\int_{t_{0}}^{t} \gamma(s) \mu(F(s)) d s
$$

If $a>0$, then:

$$
-\mathcal{M}(F(t))+\mathcal{M}(a) \leq \int_{t_{0}}^{t} \gamma(s) d s, \text { where } \mathcal{M}(x)=\int_{x}^{1} \frac{d s}{\mu(s)}
$$

If $a=0$ and $\mathcal{M}(0)=+\infty$, then $F=0$.

\section{Proof of the theorem 3.5:}

\section{First step: in which space do we work?}

Let us observe first that in view of Sobolev embedding, $q_{i} \in C\left(\mathbb{R}^{+} ; L^{\infty}\right)$. Therefore, if $\alpha$ is small enough, by embedding and continuity we get:

$$
\left|q_{i}(t, x)\right| \leq \frac{1}{2}
$$

for $x \in \mathbb{R}^{N}$ and $t$ in a small nontrivial time interval $[0, T]$.

That observation will enable us to apply proposition 2.7 to the non-linear terms involving 
$q_{i}$.

We shall further assume that $T \in(0,+\infty)$ has been chosen so small as to satisfy:

$$
C\left\|\nabla u_{2}\right\|_{L_{T}^{1}\left(B_{N, 1}^{1}\right)} \leq \log 2,
$$

for some appropriate constant $C$ whose meaning will be clear from the computations below.

To begin with, we shall prove uniqueness on the time interval $[0, T]$ by estimating $(\delta q, \delta u)$ in the following functional space:

$$
F_{T}=L^{\infty}\left([0, T] ; B_{N, \infty}^{0}\right) \times\left(L^{\infty}\left([0, T] ; B_{N, \infty}^{-1}\right) \cap \widetilde{L}_{T}^{1}\left(B_{N, \infty}^{1}\right)\right)^{N} .
$$

Indeed as explained below, in this space we can control the remainder because it is appropriate to the result of paraproduct.

\section{Why $(\delta q, \delta u)$ is in $F_{T}$ ?}

Of course, we have to state that $(\delta q, \delta u) \in F_{T}$, a fact which is not entirely obvious. We want now to show that $(\delta q, \delta u)$ belongs to $F_{T}$.

According to our assumption on $\left(q_{i}, u_{i}\right)$, the estimates of paraproduct yield $\partial_{t} q_{i} \in$ $L_{T}^{2}\left(B_{N, 1}^{0}\right)$. Therefore $\bar{q}_{i}=q_{i}-q_{0}$ belongs to $C^{\frac{1}{2}}\left([0, T], B_{N, 1}^{0}\right)$, which clearly entails by embedding $\delta q \in C\left([0, T], B_{N, \infty}^{0}\right)$.

Let $\bar{u}_{i}=u_{i}-u_{L}$ with $u_{L}$ solution to the following linear heat equation:

$$
\left\{\begin{array}{l}
\partial_{t} u_{L}-\mu \Delta u_{L}=-\bar{\delta} \nabla q_{0}+\bar{\kappa} \nabla\left(\phi * q_{0}\right), \\
u_{L}(0)=u_{0}
\end{array}\right.
$$

We obviously have $\left(\bar{u}_{i}\right)_{0}=0$ and:

$$
\partial_{t} \bar{u}_{i}-\bar{\nu} \Delta \bar{u}_{i}=-\bar{u}_{i} \cdot \nabla \bar{u}_{i}-\bar{\delta} \nabla q_{i}+\bar{\kappa} \nabla\left(\phi * q_{i}\right)+\mathcal{A}\left(\rho_{i}, q_{i}\right)+K\left(\rho_{i}, u_{i}\right) .
$$

The product and composition laws in Besov spaces insure that the right-hand side belongs to $L_{T}^{2}\left(B_{N, \infty}^{-1}\right)$ (because $\left.B_{N, 1}^{0} \times B_{N, \infty}^{0} \hookrightarrow B_{N, \infty}^{-1}\right)$ thus to $\widetilde{L}_{T}^{1}\left(B_{N, \infty}^{-1}\right)$ ) (for the last term, we use that $\bar{q}_{i} \in L_{T}^{\infty}\left(B_{N, 1}^{0}\right)$.

Now Proposition 2.9 implies that:

$$
\left.\bar{u}_{i} \in L_{T}^{\infty}\left(B_{N, \infty}^{-1}\right) \cap \widetilde{L}_{T}^{1}\left(B_{N, \infty}^{1}\right)\right) .
$$

\section{Second step: Estimates on $(\delta q, \delta u)$}

Let us turn to estimate $\delta q$. Proposition 2.8 combined with (3.37) yields for $t \leq T$ :

$$
\left.\|\delta q\|_{L_{t}^{\infty}\left(B_{N, \infty}^{0}\right)} \lesssim \int_{0}^{t}\left(\|\delta u \cdot \nabla q\|_{B_{N, \infty}^{0}}+\left\|\delta q \operatorname{div} u_{2}\right\|_{B_{N, \infty}^{0}}+\|\operatorname{div} \delta u\|_{B_{N, \infty}^{0}}\right)\right) d \tau
$$

Estimate of type $B_{p, \infty}^{\frac{N}{p}} \cap L^{\infty} \times B_{p, \infty}^{s} \hookrightarrow B_{p, \infty}^{s}$ with $s+\frac{N}{p}>0$ enables us to get the following inequality:

$$
\|\delta q\|_{L_{t}^{\infty}\left(B_{N, \infty}^{0}\right)} \lesssim \int_{0}^{t}\left(\|\delta q\|_{B_{N, \infty}^{0}}\left\|\operatorname{div} u_{2}\right\|_{B_{N, \infty}^{1} \cap L^{\infty}}+\|\delta u\|_{B_{N, \infty}^{1} \cap L^{\infty}}\left(1+\left\|q_{1}\right\|_{B_{N, 1}^{1}}\right) d \tau,\right.
$$


whence, according to Gronwall inequality, to the embedding $B_{N, 1}^{1} \hookrightarrow B_{N, \infty}^{1} \cap L^{\infty}$ and to (3.35) we get:

$$
\|\delta q\|_{L_{t}^{\infty}\left(B_{N, \infty}^{0}\right)} \lesssim\|\delta u\|_{L_{t}^{1}\left(B_{N, 1}^{1}\right)}\left(1+\left\|q_{1}\right\|_{L_{t}^{\infty}\left(B_{N, 1}^{1}\right)}\right) .
$$

Making use of (3.35) and proposition 2.4, we end up with:

$$
\|\delta q\|_{L_{t}^{1}\left(B_{N, \infty}^{0}\right)} \lesssim\|\delta u\|_{\widetilde{L}_{t}^{1}\left(B_{N, \infty}^{1}\right)} \log \left(e+\frac{\|\delta u\|_{\widetilde{L}_{t}^{1}\left(B_{N, \infty}^{0}\right)}+\|\delta u\|_{\widetilde{L}_{t}^{1}\left(B_{N, \infty}^{2}\right)}}{\left.\|\delta u\|_{\widetilde{L}_{t}^{1}\left(B_{N, \infty}^{1}\right)}\right)} .\right.
$$

Remark that:

$$
\|\delta u\|_{\widetilde{L}_{t}^{1}\left(B_{N, \infty}^{0}\right)}+\|\delta u\|_{\widetilde{L}_{t}^{1}\left(B_{N, \infty}^{2}\right)} \leq V(t)=V_{1}(t)+V_{2}(t)
$$

with:

$$
V_{i}(t)=\int_{0}^{t}\left(\left\|u_{i}(\tau)\right\|_{B_{N, 1}^{0}}+\left\|u_{i}(\tau)\right\|_{B_{N, 1}^{2}}\right) d \tau<+\infty
$$

since $\widetilde{L}_{t}^{\infty}\left(B_{N, 1}^{0}\right) \hookrightarrow \widetilde{L}_{t}^{1}\left(B_{N, 1}^{0}\right)$ for finite $t$.

We finally get:

$$
\|\delta q\|_{L_{t}^{1}\left(B_{N, \infty}^{0}\right)} \lesssim\|\delta u\|_{\widetilde{L}_{t}^{1}\left(B_{N, \infty}^{1}\right)} \log \left(e+\frac{V(t)}{\|\delta u\|_{\widetilde{L}_{t}^{1}\left(B_{N, \infty}^{1}\right)}}\right)
$$

with $V$ non-decreasing bounded function of $t \in[0,+\infty)$.

Let us now turn to the proof of estimates for $\delta u$. According to proposition 2.9, we have:

$$
\begin{gathered}
\|\delta u\|_{L_{t}^{\infty}\left(B_{N, \infty}^{-1}\right)}+\|\delta u\|_{L_{t}^{1}\left(B_{N, \infty}^{1}\right)} \lesssim\left\|u_{2} \cdot \nabla \delta u\right\|_{\widetilde{L}_{t}^{1}\left(B_{N, \infty}^{-1}\right)}+\left\|\mathcal{A}\left(q_{1}, \delta u\right)\right\|_{\widetilde{L}_{t}^{1}\left(B_{N, \infty}^{-1}\right)} \\
+\left\|\delta u \cdot \nabla u_{1}\right\|_{\widetilde{L}_{t}^{1}\left(B_{N, \infty}^{-1}\right)}+\left\|\mathcal{A}\left(\delta q, u_{2}\right)\right\|_{\widetilde{L}_{t}^{1}\left(B_{N, \infty}^{-1}\right)}+\left\|K(\delta q) \nabla q_{2}\right\|_{\widetilde{L}_{t}^{1}\left(B_{N, \infty}^{-1}\right)} \\
+\left\|K\left(q_{2}\right) \nabla \delta q\right\|_{\widetilde{L}_{t}^{1}\left(B_{N, \infty}^{-1}\right)}
\end{gathered}
$$

Let us assume that the $\alpha$ appearing in (3.35) is small enough so that the second term in the right-hand side may be absorbed by the left-hand side. $\left\|u_{2}\right\|_{\widetilde{L}_{t}^{2}\left(B_{N, 1}^{1}\right)}$ tends to 0 when $t$ goes to 0 , so if we choose $T$ small enough, the first term may also be absorbed. Using interpolation of proposition 2.4, we obtain for all $t \in[0, T]$,

$$
\|\delta u\|_{L_{t}^{\infty}\left(B_{N, \infty}^{-1}\right)}+\|\delta u\|_{L_{t}^{1}\left(B_{N, \infty}^{1}\right)} \lesssim \int_{0}^{t}\left[\left\|u_{1}\right\|_{B_{N, 1}^{2}}\|\delta u\|_{B_{N, \infty}^{-1}}+\left(1+\left\|u_{2}\right\|_{B_{N, 1}^{2}}\right)\|\delta q\|_{B_{N, \infty}^{0}}\right] d \tau
$$

Let us now plug (3.38) in the above inequality. Denoting:

$$
X(t)=\|\delta u\|_{L_{t}^{\infty}\left(B_{N, \infty}^{-1}\right)}+\|\delta u\|_{L_{t}^{1}\left(B_{N, \infty}^{1}\right)}
$$

we get for $t \leq T$,

$$
X(t) \lesssim \int_{0}^{t}\left(1+V^{\prime}(\tau)\right) X(\tau) \log \left(e+\frac{V(\tau)}{X(\tau)}\right) d \tau
$$


As:

$$
V^{\prime} \in L^{1}(0, T) \text { and } \int_{0}^{1} \frac{d r}{r \log \left(e+\frac{V(T)}{r}\right.}=+\infty,
$$

Osgood's lemma (see lemma 22) implies that $X=0$ on $[0, T]$, whence also $\delta q=0$. Standard arguments of connexity then yield uniqueness on the whole interval $[0,+\infty)$.

\section{Proof of theorem 1.2}

We will proceed similarly to the proof of the theorem 1.1. To begin with, let us observe that under the definition [1.2, system reads:

$$
\left\{\begin{array}{l}
\partial_{t} q+u \cdot \nabla u=H \\
\partial_{t} u-\bar{\nu} \Delta u=-K \\
(q, u)_{t=0}=\left(q_{0}, u_{0}\right)
\end{array}\right.
$$

with:

$$
\begin{aligned}
& H=-(1+q) \operatorname{div} u, \\
& K=G-u \cdot \nabla u+\left(P^{\prime}(\bar{\rho})+\kappa\right) \nabla \rho-\kappa \phi * \nabla \rho .
\end{aligned}
$$

As previously we can build approximate smooth solutions $\left(q^{n}, u^{n}\right)$ of (4.40) in studying the Korteweg system with a capillarity coefficient $\kappa_{n}=\frac{1}{n}$. It is convenient to split $\left(q^{n}, u^{n}\right)$ into the solution of a linear system with initial data $\left(q_{0}^{n}, u_{0}^{n}\right)$, and the discrepancy to that solution. More precisely we denote by $\left(q_{L}^{n}, u_{L}^{n}\right)$ the solution of the linearized pressure-less system on the intervall $[0, T]$ :

$$
\left\{\begin{array}{l}
\partial_{t} q_{L}^{n}+\operatorname{div} u_{L}^{n}=0, \\
\partial_{t} u_{L}^{n}-\bar{\mu} \Delta u_{L}^{n}-(\bar{\lambda}+\bar{\mu}) \nabla \operatorname{div} u_{L}^{n}=0, \\
\left(q_{L}^{n}, u_{L}^{n}\right)_{/ t=0}=\left(q_{0}^{n}, u_{0}^{n}\right),
\end{array}\right.
$$

with:

$$
\left(q_{0}^{n}, u_{0}^{n}\right)=\left(\sum_{|l| \leq n} \Delta_{l} q_{0}, \sum_{|l| \leq n} \Delta_{l} u_{0}\right)
$$

We set:

$$
\left(q^{n}, u^{n}\right)=\left(q_{L}^{n}+\bar{q}^{n}, u_{L}^{n}+\bar{u}^{n}\right)
$$

We can state now that $\left(q^{n}, \bar{u}^{n}\right)$ verifies the following linear system:

$$
\left\{\begin{array}{l}
\partial_{t} \bar{q}^{n}+\operatorname{div} \bar{u}^{n}=F^{n}, \\
\partial_{t} \bar{u}^{n}-\bar{\mu} \Delta \bar{u}^{n}-(\bar{\lambda}+\bar{\mu}) \nabla \operatorname{div} \bar{u}^{n}-\frac{1}{n} \nabla \Delta \bar{q}^{n}=G^{n}, \\
\left(\bar{q}^{n}, \bar{u}^{n}\right)_{/ t=0}=(0,0)
\end{array}\right.
$$

with:

$$
\begin{aligned}
& F^{n}=-q^{n} \operatorname{div} u^{n}, \\
& G^{n}=-u^{n} \cdot \nabla u^{n}+\mathcal{A}\left(\rho^{n}, u^{n}\right)-K\left(\rho^{n}\right) \nabla q^{n}+\left(P^{\prime}(\bar{\rho})+\kappa\right) \nabla \rho^{n}-\kappa \phi * \nabla \rho^{n} .
\end{aligned}
$$

We want show that such solution $\left(q^{n}, u^{n}\right)$ exists, in this goal we recall some theorem by R. Danchin and B. Desjardins in [19]. 
Theorem 4.6 Let $p \in\left[1,+\infty\left[\right.\right.$. Then there exists $\eta>0$ such that if $q_{0} \in B^{\frac{N}{p}}, u_{0} \in$ $\left(B^{\frac{N}{p}-1}\right)^{N}$ and:

$$
\left\|q_{0}\right\|_{B^{\frac{N}{p}}} \leq \eta
$$

then there exists $T>0$ such that system (4.40) has a unique solution $(q, u)$ in $\widetilde{E}_{T}^{p, \kappa}$.

In fact, we can extend this result to the case where the viscosity coefficients are variable. And we can show that the uniform estimates are independent of the capillarity coefficient. We can obtain the following result on our solution $\left(q^{n}, u^{n}\right)_{n \in \mathbb{N}}$.

Theorem 4.7 Let $p \in\left[1,+\infty\left[\right.\right.$. Then there exists $\eta>0$ such that if $q_{0}^{n} \in B^{\frac{N}{p}}, u_{0}^{n} \in$ $\left(B^{\frac{N}{p}-1}\right)^{N}$ and:

$$
\left\|q_{0}^{n}\right\|_{B^{\frac{N}{p}}} \leq \eta
$$

then there exists $T>0$ such that system (4.40) has a unique solution $\left(q^{n}, u^{n}\right)$ in $\widetilde{E}_{T}^{p, \frac{1}{n}}$ and $\left(q^{n}, u^{n}\right)$ are uniformly bounded in $F_{T}^{p}$.

\section{Proof:}

We recall a proposition (see [19]) on the following linearized pressure-less system:

$$
\begin{aligned}
& \partial_{t} q+\operatorname{div} u=F, \\
& \partial_{t} u-\bar{\mu} \Delta u-(\bar{\lambda}+\bar{\mu}) \nabla \operatorname{div} u-\bar{\kappa} \nabla \Delta q=G .
\end{aligned}
$$

Proposition 4.12 Let $s \in \mathbb{R}, p \in[1,+\infty], 1 \leq \rho_{1} \leq+\infty$ and $\left.\left.T \in\right] 0,+\infty\right]$. If $\left(q_{0}, u_{0}\right) \in$ $B_{p}^{s} \times\left(B_{p}^{s-1}\right)^{N}$ and $(F, G) \in \widetilde{L}_{T}^{\rho_{1}}\left(B_{p}^{s-2+\frac{2}{\rho_{1}}} \times\left(B_{p}^{s-3+\frac{2}{\rho_{1}}}\right)^{N}\right)$, then the above linear system has a unique solution $(q, u) \in \widetilde{C}_{T}\left(B_{p}^{s} \times\left(B_{p}^{s-1}\right)^{N}\right) \cap \widetilde{L}_{T}^{\rho_{1}}\left(B_{p}^{s+\frac{2}{\rho_{1}}} \times\left(B_{p}^{s-1+\frac{2}{\rho_{1}}}\right)^{N}\right)$.

Moreover for all $\rho \in\left[\rho_{1},+\infty\right]$, there exists a constant $C$ depending only on $\bar{\mu}, \bar{\lambda}, \bar{\kappa}, p$, $\rho_{1}$ and $N$ such that the following inequality holds:

$$
\begin{aligned}
\|q\|_{\widetilde{L}_{T}^{\rho}\left(B_{p}^{s+\frac{2}{\rho}}\right)}+\|u\|_{\widetilde{L}_{T}^{\rho}\left(B_{p}^{\left.s-1+\frac{2}{\rho}\right)}\right.} \leq C\left(\left\|q_{0}\right\|_{B_{p}^{s}}+\left\|u_{0}\right\|_{B_{p}^{s-1}}\right. & \\
& \left.+\|F\|_{\widetilde{L}_{T}^{\rho_{1}\left(B_{p}^{\left.s-2+\frac{2}{\rho_{1}}\right)}\right.}}+\|G\|_{\widetilde{L}_{T}^{\rho_{1}\left(B_{p}^{s-3+\frac{2}{\rho_{1}}}\right)}}\right) .
\end{aligned}
$$

Remark 2 More precisely we have:

$$
\|q\|_{\widetilde{L}_{T}^{\infty}\left(B_{p}^{s}\right)}+\kappa\|q\|_{\widetilde{L}_{T}^{1}\left(B_{p}^{s+2}\right)} \leq C\left(\left\|q_{0}\right\|_{B_{p}^{s}}+\left\|u_{0}\right\|_{B_{p}^{s-1}}+\|F\|_{\widetilde{L}_{T}^{1}\left(B_{p}^{s}\right)}+\|G\|_{\widetilde{L}_{T}^{1}\left(B_{p}^{s-1}\right)}\right) .
$$

Uniform Estimates for $\left(q^{n}, u^{n}\right)_{n \in \mathbb{N}}$

The existence of solutions in the case of general viscosity coefficient follow the same line as the proof in [19]. It suffices to solve a problem of fixed point.

Denoting by $V(t)$ the semi-group generated by system (4.41), we have:

$$
\left(q_{L}^{n}, u_{L}^{n}\right)(t)=V(t)\left(q_{0}^{n}, u_{0}^{n}\right)
$$


Let us define:

$$
\phi_{q_{L}^{n}, u_{L}^{n}}\left(\bar{q}^{n}, \bar{u}^{n}\right)=\int_{0}^{t} V(t-s)\left(F\left(q_{L}^{n}+\bar{q}^{n}, u_{L}^{n}+\bar{u}^{n}\right)(s), G\left(q_{L}^{n}+\bar{q}^{n}, u_{L}^{n}+\bar{u}^{n}\right)(s)\right) d s .
$$

where we have set:

$$
\begin{aligned}
& F(q, u)=-\operatorname{div}(q u), \\
& G(q, u)=-u \cdot \nabla u++\mathcal{A}(\rho, u)-K(\rho) \nabla q+\left(P^{\prime}(\bar{\rho})+\kappa\right) \nabla \rho-\kappa \phi * \nabla \rho .
\end{aligned}
$$

In order to prove the existence part of the theorem 4.7, ther's just have to show that $\phi_{q_{L}}^{n}, u_{L}^{n}$ has a fixed point in $F_{T}^{p}$. Since $F_{T}^{p}$ is a Banach space, we can prove that $\phi_{q_{L}^{n}, u_{L}^{n}}$ satisfies the hypothesis of Picard's theorem in a ball $\left(B(0, R)\right.$ of $F_{T}^{p}$ for sufficiently small $R$. Moreover $R$ depends only of mathematical constants. We can find a time $T$ independent of $n$ such that for all initial data verifying $\left\|q_{0}^{n}\right\|_{B^{\frac{N}{p}}} \leq \frac{R}{2}$, we have existence of solution $\left(q^{n}, u^{n}\right.$ at less on the interval $(0, T)$. The end of proof consists to verify that $\left(q^{n}, u^{n}\right)$ is uniform in $F_{T}^{p}$, it suffices to use the proposition 4.12 .

We now have builded approximated solution $\left(q^{n}, u^{n}\right)$ of system $(S W)$ and we can conclude in using technics of compactness.

\section{2) Existence of a solution}

The existence of a solution stems from compactness properties for the sequence $\left(q^{n}, u^{n}\right)_{n \in \mathbb{N}}$ an we want use some result of type Ascoli as in the proof of theorem 1.1.

Lemma 3 The sequence $\left(\partial_{t} \bar{q}^{n}, \partial_{t} \bar{u}^{n}\right)_{n \in \mathbb{N}}$ is uniformly bounded in:

$$
L^{2}\left(0, T ; \widetilde{B}^{\frac{N}{p}, \frac{N}{p}-1}\right) \times\left(L^{\alpha}\left(0, T ; \widetilde{B}^{\frac{N}{p}-1, \frac{N}{p}-2}\right)\right)^{N},
$$

for some $\alpha>1$.

\section{Proof:}

Throughout the proof, we will extensively use that $\widetilde{L}_{T}^{\rho}\left(B_{p}^{s}\right) \hookrightarrow L_{T}^{\rho}\left(B_{p}^{s}\right)$. The notation u.b will stand for uniformly bounded.

We have:

$$
\begin{aligned}
& \partial_{t} q^{n}=-u^{n} \cdot \nabla q^{n}-\left(1+q^{n}\right) \operatorname{div} u^{n}, \\
& \partial_{t} \bar{u}^{n}=-u^{n} \cdot \nabla u^{n}-q^{n} \mathcal{A}\left(\rho^{n}, \bar{u}^{n}\right)-K\left(q^{n}\right) \nabla q^{n}+\frac{1}{n} \nabla \Delta \bar{q}^{n} .
\end{aligned}
$$

We start with show that $\partial_{t} \bar{q}^{n}$ is u.b in $L^{2}\left(0, T ; \widetilde{B}_{p}^{\frac{N}{p}, \frac{N}{p}-1}\right)$.

Since $u^{n}$ is u.b in $L_{T}^{2}\left(B^{\frac{N}{p}}\right)$ and $\nabla q^{n}$ is u.b in $L_{T}^{\infty}\left(B^{\frac{N}{p}-1}\right)$, then $u^{n} \cdot \nabla q^{n}$ is u.b in $L_{T}^{2}\left(\widetilde{B}^{\frac{N}{p}, \frac{N}{p}-1}\right)$. Similar arguments enable us to conclude for the term $\left(1+q^{n}\right) \operatorname{div} u^{n}$ which is u.b in $L_{T}^{2}\left(\widetilde{B}_{p}^{\frac{N}{p}, \frac{N}{p}-1}\right)$ because $q^{n}$ is u.b in $L_{T}^{\infty}\left(B_{p}^{\frac{N}{p}}\right)$ and $\operatorname{div} u^{n}$ is u.b in $L_{T}^{2}\left(B_{p}^{\frac{N}{p}-1}\right)$.

Let us now study $\partial_{t} \bar{u}^{n+1}$. According to step one and to the definition of $u_{L}^{n}$, the term $\mathcal{A} \bar{u}^{n+1}$ is u.b in $L^{2}\left(B_{p}^{\frac{N}{p}-2}\right)$. Since $u^{n}$ is u.b in $L^{\infty}\left(B_{p}^{\frac{N}{p}-1}\right)$ and $\nabla u^{n}$ is u.b in $L^{2}\left(B_{p}^{\frac{N}{p}-1}\right)$, 
so $u^{n} \cdot \nabla u^{n}$ is u.b in $L^{2}\left(B_{p}^{\frac{N}{p}-2}\right)$ thus in $L^{2}\left(\widetilde{B}_{p}^{\frac{N}{p}-1, \frac{N}{p}-2}\right)$.

Moreover we have $q^{n}$ is u.b in $L^{\infty}\left(B_{p}^{\frac{N}{p}}\right)$ and $q^{n}$ is u.b in $L^{\infty}$, so by proposition 2.7 $\nabla K_{0}\left(q^{n}\right)$ is u.b in $L^{\infty}\left(B_{p}^{\frac{N}{p}-1}\right)$ thus in $L^{2}\left(\widetilde{B}_{p}^{\frac{N}{p}-1, \frac{N}{p}-2}\right)$. This concludes the lemma.

Now, let us turn to the proof of the existence of a solution for the system $(S W)$. We want now use some results of type Ascoli to conclude in use the properties of compactness of the lemma 3 .

According lemma 3, $\left(q^{n}, u^{n}\right)_{n \in \mathbb{N}}$ is u.b in:

$$
C^{\frac{1}{2}}\left([0, T] ; \widetilde{B}_{p}^{\frac{N}{p}, \frac{N}{p}-1}\right) \times\left(C^{1-\frac{1}{\alpha}}\left([0, T] ; \widetilde{B}_{p}^{\frac{N}{p}-1, \frac{N}{p}-2}\right)\right)^{N}
$$

thus is uniformly equicontinuous in $C\left(\left([0, T] ; \widetilde{B}_{p}^{\frac{N}{p}, \frac{N}{p}-1}\right) \times\left(\widetilde{B}_{p}^{\frac{N}{p}-1, \frac{N}{p}-2}\right)^{N}\right)$. On the other hand we have the following result of compactness, for any $\phi \in C_{0}^{\infty}\left(\mathbb{R}^{N}\right), s \in \mathbb{R}, \delta>0$ the application $u \rightarrow \phi u$ is compact from $B_{p}^{s}$ to $\widetilde{B}_{p}^{s, s-\delta}$. Applying Ascoli's theorem, we infer that up to an extraction $\left(q^{n}, u^{n}\right)_{n \in \mathbb{N}}$ converges in $\mathcal{D}^{\prime}\left([0, T] \times \mathbb{R}^{N}\right)$ to a limit $(\bar{q}, \bar{u})$ which belongs to:

$$
C^{\frac{1}{2}}\left([0, T] ; \widetilde{B}_{p}^{\frac{N}{p}, \frac{N}{p}-1}\right) \times\left(C^{1-\frac{1}{\alpha}}\left([0, T] ; \widetilde{B}_{p}^{\frac{N}{p}-1, \frac{N}{p}-2}\right)\right)^{N}
$$

Let $(q, u)=(\bar{q}, \bar{u})+\left(q_{0}, u_{L}\right)$. Using again uniform estimates of step one and proceeding as, we gather that $(q, u)$ solves $(S W)$ and belongs to:

$$
\bar{\rho}+\widetilde{L}_{T}^{\infty}\left(\widetilde{B}_{p}^{\frac{N}{p}, \frac{N}{p}-1}\right) \times\left(\widetilde{L}_{T}^{1}\left(B_{p}^{\frac{N}{p}+1}\right) \cap \widetilde{L}_{T}^{\infty}\left(B_{p}^{\frac{N}{p}-1}\right)\right)^{N} .
$$

Applying proposition, we get the continuity results:

$$
\rho-\bar{\rho} \in C\left([0, T], \widetilde{B}_{p}^{\frac{N}{p}, \frac{N}{p}-1}\right), \quad u \in C\left([0, T], B_{p}^{\frac{N}{p}-1}\right) .
$$

\section{Proof of theorem 1.3}

In this section, we consider the case when the initial density belongs to $\bar{\rho}+\widetilde{B}^{\frac{N}{p}, \frac{N}{p}+\varepsilon}$ and satisfies $0<\bar{\rho} \leq \rho_{0}$. We consider again the study of a approximate sequence verifying (4.40) and we proceed as previously.

\section{Construction of approximate solutions}

We consider the following system:

$$
\begin{aligned}
& \partial_{t} q^{n+1}+u^{n} \cdot \nabla q^{n+1}=q^{n} \operatorname{div} u^{n}, \\
& \partial_{t} u^{n+1}+u^{n} \cdot \nabla u^{n+1}-\mathcal{A}\left(u^{n+1}\right)+\nabla q^{n+1}=G^{n},
\end{aligned}
$$

with:

$$
G^{n}=\mathcal{A}\left(u^{n}\right)-\mathcal{A}\left(\rho^{n}, u^{n}\right)+K\left(\rho^{n}\right) \nabla q^{n} .
$$


Uniform estimates for $\left(q^{n}, u^{n}\right)_{n \in \mathbb{N}}$

Let us show that the sequence $\left(q^{n}, u^{n}\right)_{n \in \mathbb{N}}$ is uniformly bounded in provided that $T$ and $\eta$ have been chosen small enough.

Let us remark first that according to proposition, there exists a universal condition $K$ such that for all $n \in \mathbb{N}$, we have:

$$
\left\|u_{L}^{n}\right\|_{\widetilde{L}_{T}^{\infty}\left(B^{\frac{N}{p}}\right)} \leq U_{0}
$$

with $U_{0}=K\left\|u_{0}\right\|_{B^{\frac{N}{2}}}$,

$$
\left\|u_{L}^{n}\right\|_{\widetilde{L}_{T}^{1}\left(B^{\frac{N}{p}+1}\right)} \leq K \sum_{q \in \mathbb{Z}} 2^{q\left(\frac{N}{p}-1\right)}\left\|\Delta_{q} u_{0}\right\|
$$

Suppose that $\left\|q_{0}\right\|_{B^{\frac{N}{p}}} \leq \eta$ for a small constant $\eta$ and let $C_{0}=1+\left\|q_{0}\right\|_{B^{\frac{N}{p}}}$. According to , we can choose a positive time $T$ such that the following property holds for all $n \in \mathbb{N}$ :

$$
\left\|u_{L}^{n}\right\|_{\widetilde{L}_{T}^{\infty}\left(B^{\frac{N}{p}}\right)} \leq U_{0}, \quad \text { and } \quad\left\|u_{L}^{n}\right\|_{\widetilde{L}_{T}^{r}\left(B^{\frac{N}{p}+1}\right)} \leq \eta^{\frac{2}{r}} U_{0}^{1-\frac{1}{r}}
$$

Let us now show by induction that the following estimates are satisfied:

$$
\begin{aligned}
& \left\|q^{n}\right\|_{\widetilde{L}_{T}^{\infty}\left(B^{\frac{N}{p}}\right)} \leq \sqrt{\eta} \\
& \left\|\bar{u}^{n}\right\|_{\widetilde{L}_{T}^{\infty}\left(B^{\frac{N}{p}-1}\right)}+\left\|\bar{u}^{n}\right\|_{\widetilde{L}_{T}^{1}\left(B^{\frac{N}{p}+1}\right)} \leq \eta
\end{aligned}
$$

From now, we suppose that $\eta \leq\left(2 C_{1}\right)^{-2}$ where $C_{1}$ is the norm of the injection $B^{\frac{N}{p}} \hookrightarrow L^{\infty}$. This ensures us that the following inequality is satisfied:

$$
\frac{1}{2} \leq 1+q^{n} \leq \frac{3}{2}
$$

According to proposition, we have:

$$
\left\|q^{n+1}\right\|_{\widetilde{L}_{T}^{\infty}\left(B^{\frac{N}{p}}\right)} \leq \exp { }^{C\left\|u^{n}\right\|_{L_{T}^{1}\left(B^{\frac{N}{p}+1}\right)}}\left(\left\|q_{0}\right\|_{B^{\frac{N}{p}}}+\left\|F^{n}\right\|_{L_{T}^{1}\left(B^{\frac{N}{p}}\right)}\right)
$$

Moreover we have by Proposition:

$$
\left\|u^{n}\right\|_{\widetilde{L}_{T}^{\infty}\left(B^{\frac{N}{p}}\right)} \leq \exp { }^{C\left\|u^{n}\right\|_{L_{T}^{1}\left(B^{\frac{N}{p}+1}\right)}}\left(\left\|q_{0}\right\|_{B^{\frac{N}{p}}}+\left\|F^{n}\right\|_{L_{T}^{1}\left(B^{\frac{N}{p}}\right)}\right)
$$

whence according to proposition:

$$
\left\|F_{n}\right\|_{L_{T}^{1}\left(B^{\frac{N}{p}}\right)} \lesssim T^{1-\frac{1}{r_{1}}}\left(1+\left\|q^{n}\right\|_{L_{T}^{\infty}\left(B^{\frac{N}{p}}\right)}\right)\left\|\operatorname{div} u^{n}\right\|_{L_{T}^{r_{1}\left(B^{\frac{N}{p}}\right)}} \lesssim\left(1+U_{0}\right)^{\frac{1}{2}} \eta
$$

and we get finally:

$$
\left\|q^{n}\right\|_{\widetilde{L}_{T}^{\infty}\left(B^{\frac{N}{p}}\right)} \leq\left(1+U_{0}\right)^{\frac{1}{2}} \eta \exp ^{C\left(1+U_{0}\right)^{\frac{1}{2}} \eta}
$$


Obviously if $\eta$ has been chosen small enough then $q^{n+1}$ satisfies the estimate in $\left(\mathcal{P}_{n+1}\right)$. Applying proposition to the second equation of yields:

$$
\begin{aligned}
\left\|\bar{u}^{n+1}\right\|_{\widetilde{L}_{T}^{\infty}\left(B^{\frac{N}{p}}\right)}+\left\|\bar{u}^{n+1}\right\|_{L_{T}^{1}\left(B^{\frac{N}{p}+1}\right)} \lesssim\left\|u^{n} \cdot \nabla u^{n}\right\|_{L_{T}^{1}\left(B^{\frac{N}{p}-1}\right)} & \\
& \left\|K_{0}\left(q^{n}\right) \nabla q^{n}\right\|_{L_{T}^{1}\left(B^{\frac{N}{p}-1}\right)} \\
& +\left\|\mathcal{A}\left(q^{n}, u^{n}\right)\right\|_{L_{T}^{1}\left(B^{\frac{N}{p}-1}\right)}
\end{aligned}
$$

We now use proposition 2.7 as in the proof of ?? to conclude.

\section{Existence of a solution}

We can now easily show that $\left(q^{n}, u^{n}\right)$ is a Cauchy sequel in our space $F_{T}$ of uniqueness and so $\left(q_{n}, u_{n}\right) \rightarrow(q, u)$ in $F_{T}$.

It rests to verify by compactness that $(q, u)$ is a solution of the system $(S W)$.

\section{References}

[1] H. Bahouri and J.-Y. Chemin, Équations d'ondes quasilinéaires et estimation de Strichartz, Amer. J. Mathematics 121 (1999) 1337-1377.

[2] J.-M. Bony, Calcul symbolique et propagation des singularités pour les équations aux dérivées partielles non linéaires, Annales Scientifiques de l'école Normale Supérieure 14 (1981) 209-246.

[3] G. Bourdaud, Réalisations des espaces de Besov homogènes, Arkiv fur Mathematik 26 (1998) 41-54.

[4] D. Bresch and B. Desjardins, Existence of global weak solutions for a 2D Viscous shallow water equations and convergence to the quasi-geostrophic model. Comm. Math. Phys., 238(1-2): 211-223, 2003.

[5] D. Bresch and B. Desjardins, Existence of global weak solutions to the Navier-Stokes equations for viscous compressible and heat conducting fluids, to appear.

[6] D. Bresch and B. Desjardins, Some diffusive capillary models of Koretweg type. C. R. Math. Acad. Sci. Paris, Section Mécanique, 332(11):881-886, 2004.

[7] D. Bresch, B. Desjardins and C.-K. Lin, On some compressible fluid models: Korteweg, lubrification and shallow water systems. Comm. Partial Differential Equations, $28(3-4): 843-868,2003$.

[8] J.-Y. Chemin, Théorèmes d'unicité pour le système de Navier-Stokes tridimensionnel, J.d'Analyse Math. 77 (1999) 27-50.

[9] J.-Y. Chemin, About Navier-Stokes system, Prépublication du Laboratoire d'Analyse Numérique de Paris 6 R96023 (1996).

[10] J.-Y. Chemin and N. Lerner, Flot de champs de vecteurs non lipschitziens et équations de Navier-Stokes, J.Differential Equations 121 (1992) 314-328. 
[11] H. J. Choe and H. Kim, Strong solution of the Navier-Stokes equations for isentropic compressible fluids, J. Differential Equations 190 (2003), 504-523.

[12] H. J. Choe and H. Kim, Strong solution of the Navier-Stokes equations for nonhomogeneous incompressible fluids, Math. Meth. Appl. Sci. 28 (2005), 1-28.

[13] F. Coquel, D. Diehl, C. Merkle and C. Rohde, Sharp and diffuse interface methods for phase transition problems in liquid-vapour flows.

[14] R. Danchin, Fourier analysis method for PDE's, Preprint Novembre 2005.

[15] R. Danchin, Global Existence in Critical Spaces for Flows of Compressible Viscous and Heat-Conductive Gases, Arch.Rational Mech.Anal.160 (2001) 1-39

[16] Danchin.R, Density-dependent incompressible viscous fluids in critical spaces. Proc. Roy. Soc. Edimburgh Sect. A, 133(6): 1311-1334, 2003.

[17] Danchin.R, A few remarks on the Camassa-Holm equation, Differential and Integral Equations 14 (2001), 953-988.

[18] Danchin.R, Local Theory in critical Spaces for Compressible Viscous and HeatConductive Gases,Communication in Partial Differential Equations 26 (78),1183-1233 (2001)

[19] R. Danchin and B. Desjardins, Existence of solutions for compressible fluid models of Korteweg type, Annales de l'IHP,Analyse non linéaire 18,97-133 (2001)

[20] E. Feireisl, Dynmamics of Viscous Compressible Fluids-Oxford Lecture Series in Mathematics and its Applications-26.

[21] E. Feireisl. Compressible Navier-Stokes equations with a non-monotone pressure law. J. Differential Equations, 184(1):97-108, 2002.

[22] E. Feireisl. On the motion of a viscous, compressible, and heat conducting equation. Indiana Univ. Math. J., 53(6):1705-1738, 2004.

[23] E. Feireisl, Antonín Novotný, and Hana Petzeltová. On the existence of globally defined weak solutions to the Navier-Stokes equations. J. Math. Fluid Mech., 3(4):358$392,2001$.

[24] David Hoff. Global existence for 1D, compressible, isentropic Navier-Stokes equations with large initial data. Trans. Amer. Math. Soc, 303(1):169-181, 1987.

[25] David Hoff. Discontinuous solutions of the Navier-Stokes equations for multidimensional flows of the heat conducting fluids. Arch. Rational Mech. Anal., 139, (1997), P. 303-354.

[26] David Hoff. Global solutions of the Navier-Stokes equations for multidimensional compressible flow with discontinuous initial data. J. Differential Equations, 120(1):215254, 1995. 
[27] David Hoff. Strong convergence to global solutions for multidimensional flows of compressible, viscous fluids with polytropic equations of state and discontinuous initial data. Arch. Rational Mech. Anal., 132(1):1-14, 1995.

[28] David Hoff and Kevin Zumbrum. Multi-dimensional diffusion waves for the NavierStokes equations of compressible flow, Indiana University Mathematics Journal, 1995, $44,603-676$.

[29] Song Jiang and Ping Zhang. Axisymetrics solutions of the 3D Navier-Stokes equations for compressible isentropic fluids. J. Math. Pures Appl. (9), 82(8):949-973, 2003.

[30] A. V. Kazhikov. The equation of potential flows of a compressible viscous fluid for small Reynolds numbers: existence, uniqueness and stabilization of solutions. Sibirsk. Mat. Zh., 34 (1993), no. 3, p. 70-80.

[31] A. V. Kazhikov and V. V. Shelukhin. Unique global solution with respect to time of initial-boundary value problems for one- dimensional equations of a viscous gas. Prikl. Mat. Meh., 41(2):282-291, 1977.

[32] P.-L. Lions, Mathematical Topics in Fluid Mechanics, Vol 2, Compressible models, Oxford University Press (1998)

[33] Akitaka Matsumura and Takaaki Nishida. The initial value problem for the equations of motion of compressible viscous and heat-conductive fluids. Proc. Japan Acad. Ser. A Math. Sci, 55(9):337-342, 1979.

[34] A.Mellet and A.Vasseur, On the isentropic compressible Navier-Stokes equation, Arxiv preprint math.AP/0511210, 2005 - arxiv.org

[35] J. Nash, Le problème de Cauchy pour les équations différentielles d'un fluide général, Bulletin de la Société Mathématique de France, 1962, 90, 487-497.

[36] C. Rohde, On local and non-local Navier-Stokes-Korteweg systems for liquidvapour phase transitions. Technical report, Math. Institut, Albert-Ludwigs-Universität Freiburg , 2004. Preprint.

[37] T. Runst and W. Sickel: Sobolev spaces of fractional order, Nemytskij operators, and nonlinear partial differential equations. de Gruyter Series in Nonlinear Analysis and Applications, 3. Walter de Gruyter and Co., Berlin (1996)

[38] Denis Serre. Solutions faibles globales des équations de Navier-Stokes pour un fluide compressible., 303(13):639-642, 1986

[39] V.A. Solonnikov. Estimates for solutions of nonstationary Navier-Stokes systems. Zap. Nauchn. Sem. LOMI, 38, (1973), p.153-231; J. Soviet Math. 8, (1977), p. 467529.

[40] V. Valli, W. Zajaczkowski. Navier-Stokes equations for compressible fluids: global existence and qualitative properties of the solutions in the general case. Commun. Math. Phys., 103 (1986) no 2., p. 259-296. 
[41] Weike Wang and Chao-Jiang Xu. The Cauchy problem for viscous shallow water equations. Rev. Mat. Iberoamericana 21, no. 1 (2005), 1-24. 